\title{
Convergent antibody responses to SARS-CoV-2 in convalescent individuals
}

https://doi.org/10.1038/s41586-020-2456-9

Received: 3 May 2020

Accepted: 12 June 2020

Published online: 18 June 2020

Check for updates

\author{
Davide F. Robbiani ${ }^{1,9,10 凶}$, Christian Gaebler ${ }^{1,10}$, Frauke Muecksch ${ }^{2,10}$, Julio C. C. Lorenzi ${ }^{1,10}$, \\ Zijun Wang ${ }^{1,10}$, Alice Cho ${ }^{1,10}$, Marianna Agudelo ${ }^{1,10}$, Christopher O. Barnes ${ }^{3,10}$, \\ Anna Gazumyan ${ }^{1,10}$, Shlomo Finkin ${ }^{1,10}$, Thomas Hägglöf ${ }^{1,10}$, Thiago Y. Oliveira ${ }^{1,10}$, \\ Charlotte Viant ${ }^{1,10}$, Arlene Hurley ${ }^{4}$, Hans-Heinrich Hoffmann ${ }^{5}$, Katrina G. Millard', \\ Rhonda G. Kost ${ }^{6}$, Melissa Cipolla', Kristie Gordon', Filippo Bianchini', Spencer T. Chen', \\ Victor Ramos', Roshni Patel', Juan Dizon', Irina Shimeliovich', Pilar Mendoza', \\ Harald Hartweger', Lilian Nogueira', Maggi Pack', Jill Horowitz', Fabian Schmidt' \\ Yiska Weisblum ${ }^{2}$, Eleftherios Michailidis ${ }^{5}$, Alison W. Ashbrook ${ }^{5}$, Eric Waltari ${ }^{7}$, John E. Pak ${ }^{7}$, \\ Kathryn E. Huey-Tubman ${ }^{3}$, Nicholas Koranda ${ }^{3}$, Pauline R. Hoffman ${ }^{3}$, Anthony P. West Jr ${ }^{3}$, \\ Charles M. Rice ${ }^{5}$, Theodora Hatziioannou ${ }^{2}$, Pamela J. Bjorkman ${ }^{3 凶}$, Paul D. Bieniasz $^{2,8} \bowtie$, \\ Marina Caskey ${ }^{1 凶}$ \& Michel C. Nussenzweig $^{1,8 凶}$
}

During the coronavirus disease-2019 (COVID-19) pandemic, severe acute respiratory syndrome-related coronavirus-2 (SARS-CoV-2) has led to the infection of millions of people and has claimed hundreds of thousands of lives. The entry of the virus into cells depends on the receptor-binding domain (RBD) of the spike (S) protein of SARS-CoV-2. Although there is currently no vaccine, it is likely that antibodies will be essential for protection. However, little is known about the human antibody response to SARS-CoV-2 ${ }^{1-5}$. Here we report on 149 COVID-19-convalescent individuals. Plasma samples collected an average of 39 days after the onset of symptoms had variable half-maximal pseudovirus neutralizing titres; titres were less than 50 in $33 \%$ of samples, below 1,000 in $79 \%$ of samples and only $1 \%$ of samples had titres above 5,000 . Antibody sequencing revealed the expansion of clones of RBD-specific memory B cells that expressed closely related antibodies in different individuals. Despite low plasma titres, antibodies to three distinct epitopes on the RBD neutralized the virus with half-maximal inhibitory concentrations $\left(\mathrm{IC}_{50}\right.$ values) as low as $2 \mathrm{ng} \mathrm{ml}^{-1}$. In conclusion, most convalescent plasma samples obtained from individuals who recover from COVID-19 do not contain high levels of neutralizing activity.

Nevertheless, rare but recurring RBD-specific antibodies with potent antiviral activity were found in all individuals tested, suggesting that a vaccine designed to elicit such antibodies could be broadly effective.
Between 1 April and 8 May 2020,157 eligible participants were enrolled in the study. Of these, $111(70.7 \%)$ were individuals who had a SARS-CoV-2 infection, as confirmed by PCR with reverse transcription (RT-PCR) (cases), and 46 (29.3\%) were close contacts of individuals diagnosed with SARS-CoV-2 infection (contacts). Although inclusion criteria allowed for enrolment of asymptomatic participants, eight contacts who did not develop symptoms were excluded from further analyses. The 149 cases and contacts were free of symptoms that are suggestive of COVID-19 for at least 14 days at the time of sample collection. Participant demographics and clinical characteristics are shown in Extended Data Fig. 1a and Supplementary Tables 1, 2. Only one individual who tested positive for SARS-CoV-2 infection by RT-PCR remained asymptomatic.
The other 148 participants reported symptoms that were suggestive of COVID-19 with a mean time of onset of symptoms of approximately 39 days (range, 17-67 days) before sample collection. In this cohort, symptoms lasted for an average of 12 days (0-35 days), and 11 (7\%) of the participants were hospitalized. The most common symptoms were fever (83.9\%), fatigue (71.1\%), cough (62.4\%) and myalgia (61.7\%), whereas baseline comorbidities were infrequent (10.7\%) (Supplementary Tables 1,2). There were no significant differences in duration or severity (Methods) of symptoms, or in the time from onset of symptoms to sample collection between genders or between cases and contacts. There was no age difference between women and men in our cohort (Extended Data Fig. 1).

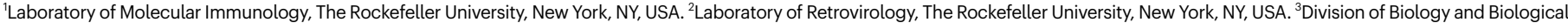

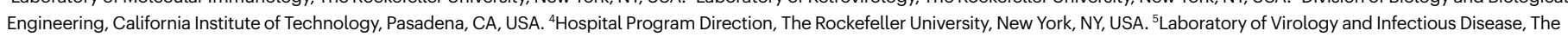
Rockefeller University, New York, NY, USA. ${ }^{6}$ Center for Clinical Translational Science, The Rockefeller University, New York, NY, USA. ${ }^{7}$ Chan Zuckerberg Biohub, San Francisco, CA, USA. ${ }^{8}$ Howard

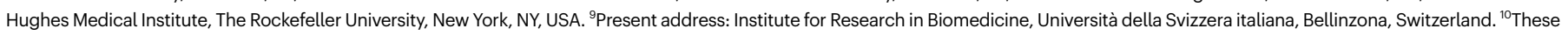

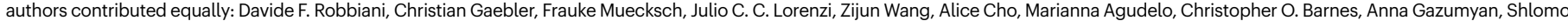

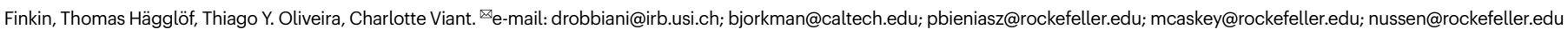



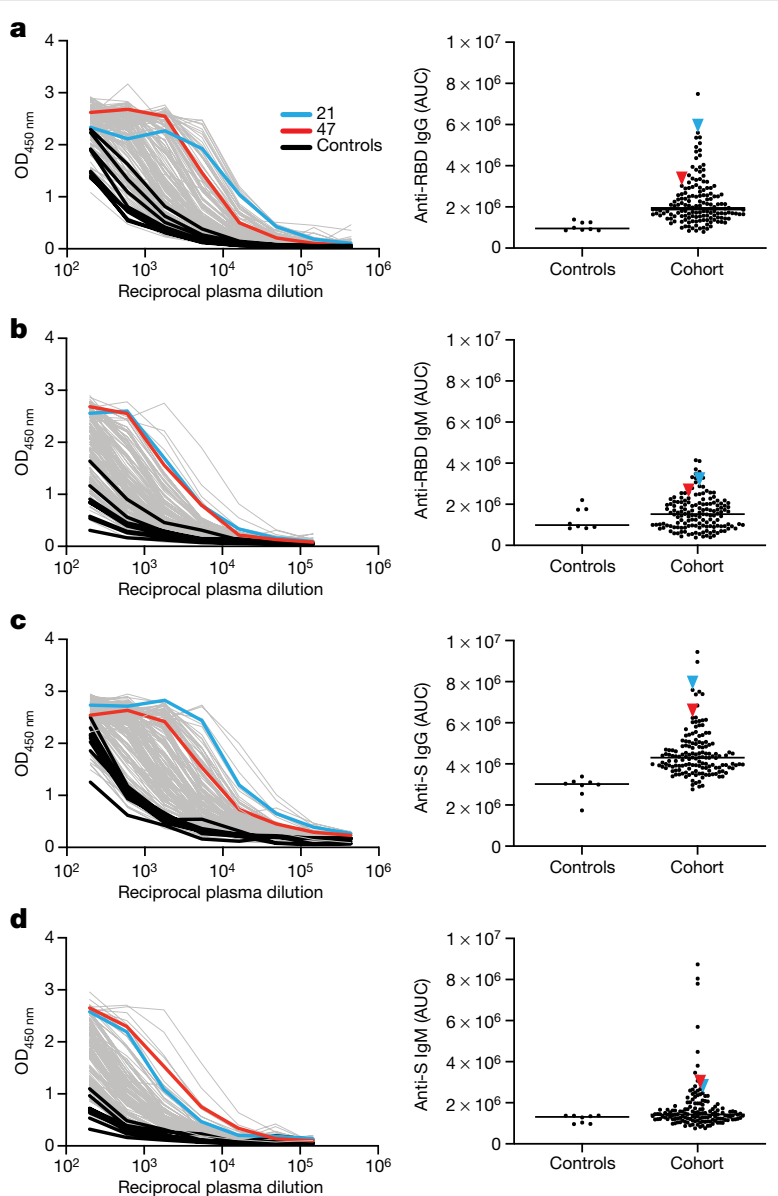

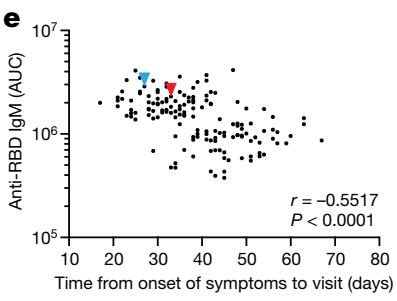

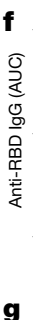
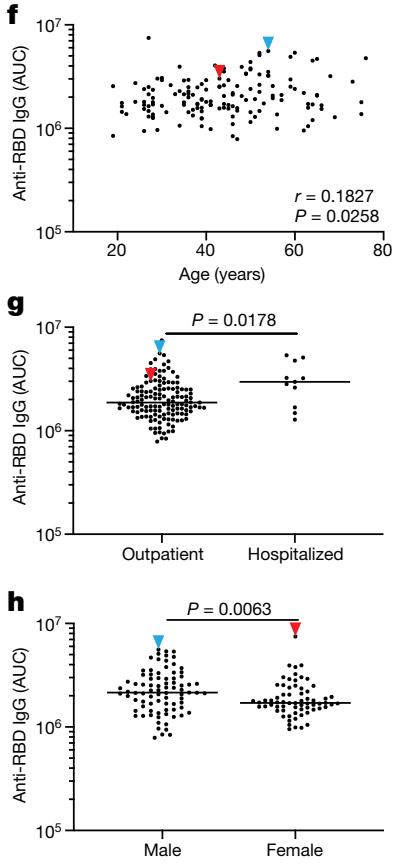

Fig. 1 | Plasma antibodies against SARS-CoV-2. a-d, Results of ELISAs measuring plasma reactivity to $\operatorname{RBD}(\mathbf{a}, \mathbf{b})$ and S protein (c, d). a, Anti-RBD IgG. b, Anti-RBD IgM.c, Anti-S IgG. d, Anti-S IgM. Left, optical density at $450 \mathrm{~nm}$ $\left(\mathrm{OD}_{450 \mathrm{~nm}}\right)$ for the indicated reciprocal plasma dilutions. Right, the normalized area under the curve (AUC) for the 8 controls and 149 individuals in the cohort. Negative controls are shown in black; individuals 21 and 47 are shown as blue and red lines or arrowheads, respectively. e, Time between symptom onset and time of sample collection in days is plotted against the normalized AUC for IgM

Plasma samples were tested for binding to the SARS-CoV-2 RBD and trimeric $S$ proteins by a validated enzyme-linked immunosorbent assay (ELISA) using anti-IgG or anti-IgM secondary antibodies for detection $^{6,7}$ (Fig. 1, Extended Data Figs. 2,3 and Supplementary Table 1). Eight independent negative controls and a positive control plasma sample from participant 21 (COV21) were included for normalization of the area under the curve (AUC) in all experiments. Overall, $78 \%$ and $70 \%$ of the tested plasma samples showed anti-RBD and anti-S IgG AUCs that were at least two standard deviations above the control (Fig. 1a, b). By contrast, only $15 \%$ and $34 \%$ of the plasma samples showed IgM responses to anti-RBD and anti-S, respectively, that were at least two standard deviations above control (Fig. 1c, d). There was no positive correlation between anti-RBD or anti-S IgG or IgM levels and the duration of symptoms or the timing of sample collection relative to the onset of symptoms (Fig. 1e and Extended Data Fig. $3 a-c, g-j$ ). By contrast, as might be expected, anti-RBD IgM titres were negatively correlated with the duration of symptoms and the timing of sample collection (Fig. 1e and Extended Data Fig. 3h). Anti-RBD IgG levels were moderately correlated with age and the severity of symptoms including hospitalization (Fig. If, g and Extended Data Fig. 3k). Notably, women had lower anti-RBD and anti-S IgG titres than men (Fig. 1h and Extended Data Fig. 2f).

To measure the neutralizing activity in convalescent plasma samples, we used HIV-1-based virions that carried a nanoluc luciferase reporter, which were pseudotyped with the SARS-CoV-2S protein (SARS-CoV-2 binding to RBD. $r=-0.5517, P<0.0001 . f$, Participant age in years is plotted against normalized AUC for IgG binding to RBD. $r=0.1827$ and $P=0.0258$. The $r$ and $P$ values in e and $\mathbf{f}$ were determined by two-tailed Spearman's correlations. g, Normalized AUC of anti-RBD IgG ELISA for outpatients $(n=138)$ and hospitalized individuals $(n=11) . P=0.0178 . \mathbf{h}$, Normalized AUC of anti-RBD IgG ELISA for men $(n=83)$ and women $(n=66) . P=0.0063$. For $\mathbf{g}$ and $\mathbf{h}$, horizontal bars indicate median values. Statistical significance was determined using two-tailed Mann-Whitney $U$-tests.

pseudovirus; Fig. 2, Methods and Extended Data Fig. 4). Negative (historical samples) and positive (COV21) controls were included in all experiments. The overall level of neutralizing activity in the cohort, as measured by the half-maximal neutralizing titre $\left(\mathrm{NT}_{50}\right)$, was generally low; $\mathrm{NT}_{50}$ values were less than 50 in $33 \%$ of samples and below 1,000 in $79 \%$ of samples (Fig. 2a, b). The geometric mean $\mathrm{NT}_{50}$ was 121 (arithmetic mean $=714$ ), and only 2 individuals reached $\mathrm{NT}_{50}$ values above 5,000 (Fig. 2a, b and Supplementary Table 1).

Notably, levels of anti-RBD and anti-S IgG antibodies correlated strongly with $\mathrm{NT}_{50}$ values (Fig. 2c, d). Neutralizing activity also correlated with age, the duration of symptoms and the severity of symptoms (Extended Data Fig. 5). Consistent with this observation, hospitalized individuals with a longer duration of symptoms showed slightly higher average levels of neutralizing activity than individuals who were not hospitalized ( $P=0.0495)$ (Fig. 2e). Finally, we observed a significant difference in neutralizing activity between men and women $(P=0.0031)$ (Fig. 2f). The difference between men and women was consistent with higher anti-RBD and anti-S IgG titres in men, and could not be attributed to age, severity of symptoms, timing of sample collection relative to the onset or duration of symptoms (Fig. 1h and Extended Data Figs. $1 b-e, 2 f)$.

To determine the nature of the antibodies elicited by SARS-CoV-2 infection, we used flow cytometry to isolate individual B lymphocytes that carried receptors that bound to the RBD from the blood 

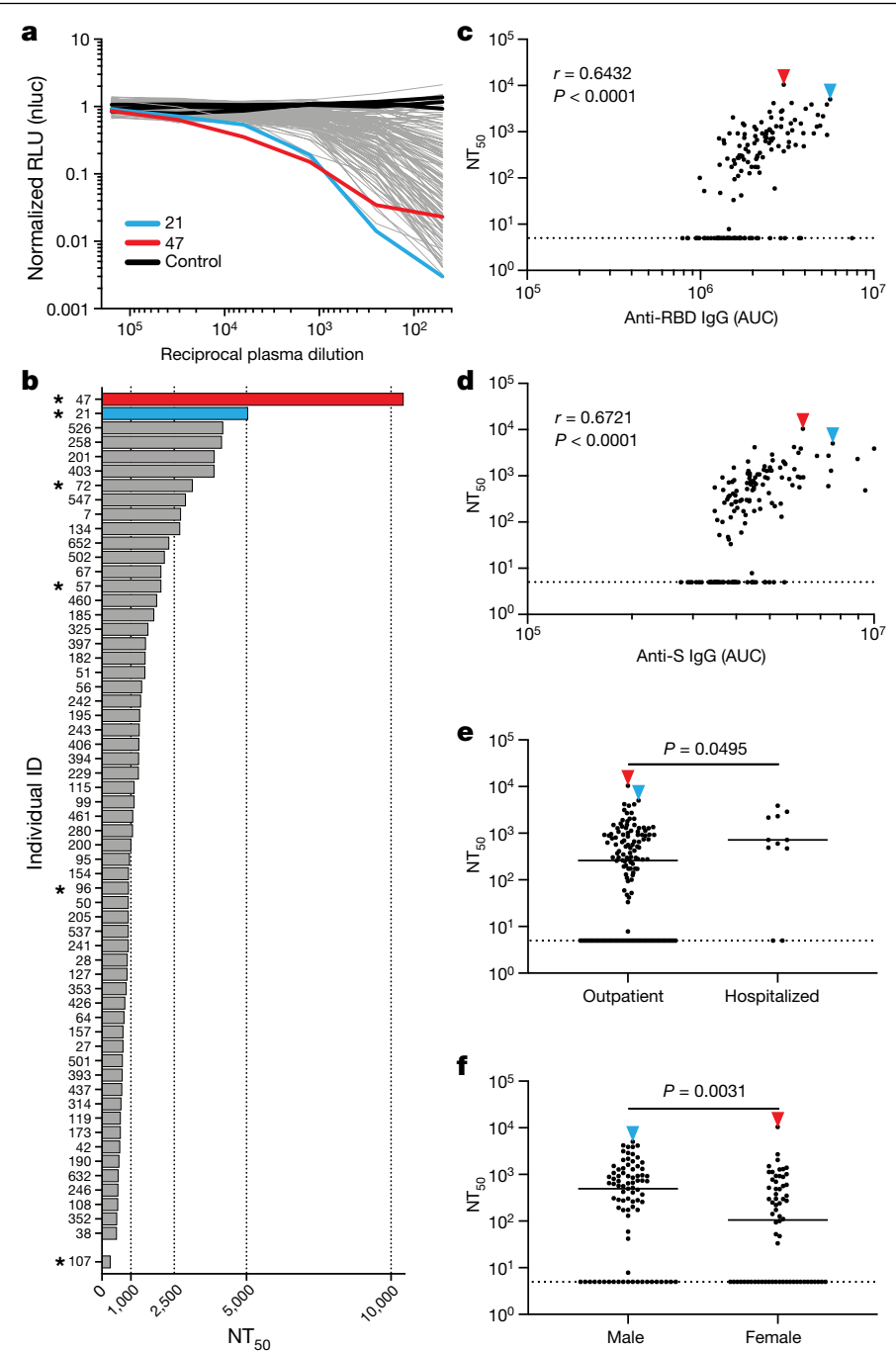

Fig. 2 Neutralization of SARS-CoV-2 pseudovirus by plasma. a, The normalized relative luminescence values (RLU) for cell lysates of $293 \mathrm{~T}_{\mathrm{ACE} 2}$ cells $48 \mathrm{~h}$ after infection with nanoluc-expressing SARS-CoV-2 pseudovirus in the presence of increasing concentrations of plasma derived from 149 participants (grey; except individuals 21 and 47 , for which data are shown in blue and red, respectively) and 3 negative controls (black lines). Data are the mean of duplicates; representative of two independent experiments. b. Ranked average half-maximal inhibitory plasma neutralizing titre $\left(\mathrm{NT}_{50}\right)$ for the 59 out of 149 individuals with $\mathrm{NT}_{50}>500$ and individual 107. Asterisks indicate donors from whom antibody sequences were derived. c, Normalized AUC for anti-RBD IgG ELISA plotted against $\mathrm{NT}_{50}$ values. $r=0.6432, P<0.0001$. d, Normalized AUC for anti-S IgG ELISA plotted against $\mathrm{NT}_{50}$ values. $r=0.6721, P<0.0001$. The $r$ and $P$ values in $\mathbf{c}$ and $\mathbf{d}$ were determined by two-tailed Spearman's correlations. e, $\mathrm{NT}_{50}$ values for outpatients $(n=138)$ and hospitalized individuals $(n=11)$. $P=0.0495 . \mathrm{f}, \mathrm{NT}_{50}$ values for men $(n=83)$ and women $(n=66)$ in the cohort. $P=0.0031$. Statistical significance in $\mathbf{e}$ and $\mathbf{f}$ was determined using two-tailed Mann-Whitney $U$-tests and horizontal bars indicate median values. Dotted lines in $\mathbf{c}-\mathbf{f}\left(\mathrm{NT}_{50}=5\right)$ represent the lower limit of detection. Samples with neutralizing titres below 50 were plotted at the lower limit of detection.

of six selected individuals, including the two samples with top neutralizing activity and four samples with high-to-intermediate neutralizing activity (Fig. 3). The frequency of antigen-specific B cells, identified by their ability to bind to both phycoerythrin (PE)- and AlexaFluor-647-labelled RBD, ranged from 0.07 to $0.005 \%$ of all circulating B cells in COVID-19-convalescent individuals, whereas they were undetectable in pre-COVID-19 control samples (Fig. 3a and Extended Data Fig. 6). We obtained 534 paired IgG heavy and light chain (IGH and IGL) sequences by RT-PCR from individual RBD-binding B cells from the 6 convalescent individuals (Methods and Supplementary Table 3). When compared to the human antibody repertoire, several IGHV and IGLV genes were significantly overrepresented (Extended Data Fig. 7). The average number of nucleotide mutations in V genes for IGH and IGL was 4.2 and 2.8, respectively (Extended Data Fig. 8), which is lower than in antibodies cloned from individuals with chronic infections such as hepatitis B or HIV-1, and similar to antibodies derived from individuals with a primary malaria infection or from non-antigen-enriched circulating IgG memory cells ${ }^{8-11}$. Among other antibody features, IGH CDR3 length was indistinguishable from the reported norm and hydrophobicity was below average ${ }^{12}$ (Extended Data Fig. 8).

As is the case with other human pathogens, there were expanded clones of viral antigen-binding B cells in all tested individuals convalescent after COVID-19 (Fig. 3b, c and Methods). Overall, 32.2\% of the recovered IGH and IGL sequences were from clonally expanded B cells (range, 21.8-57.4\% across individuals) (Fig. 3b). Antibodies that shared specific combinations of $I G H V$ and $I G L V$ genes in different individuals comprised $14 \%$ of all the clonal sequences (Fig. 3b, c). Notably, the amino acid sequences of some antibodies found in different individuals were nearly identical (Fig. 3d). For example, antibodies expressed by clonally expanded B cells with IGHV1-58/IGKV3-20 and IGHV3-30-3/ IGKV1-39 were found repeatedly in different individuals and had amino acid sequence identities of up to $99 \%$ and $92 \%$, respectively (Fig. $3 \mathrm{~d}$ and Supplementary Table 4). We conclude that the IgG memory response to the SARS-CoV-2 RBD is rich in recurrent and clonally expanded antibody sequences.

To examine the binding properties of anti-SARS-CoV-2 antibodies, we expressed 94 representative antibodies, 67 from clones and 27 from singlets (Supplementary Table 5). ELISAs showed that $95 \%$ (89 out of 94) of the antibodies tested including clonal and unique sequences bound to the SARS-CoV-2 RBD with an average half-maximal effective concentration $\left(\mathrm{EC}_{50}\right.$ ) of $6.9 \mathrm{ng} \mathrm{ml}^{-1}$ (Fig. 4a and Extended Data Fig. 9a). A fraction of these (7 out of 77 that were tested, or $9 \%$ ) cross-reacted with the RBD of SARS-CoV with $\mathrm{EC}_{50}$ values below $1 \mu \mathrm{g} \mathrm{ml}^{-1}$ (Extended Data Fig. 9b, c). No significant cross-reactivity was noted to the RBDs of MERS, HCoV-OC43, HCoV-229E or HCoV-NL63.

To determine whether the monoclonal antibodies had neutralizing activity, we tested them against the SARS-CoV-2 pseudovirus (Fig. 4 and Supplementary Table 6). Among 89 RBD-binding antibodies tested, we found 52 that neutralized SARS-CoV-2 pseudovirus with $\mathrm{IC}_{50}$ values ranging from 3 to $709 \mathrm{ng} \mathrm{ml}^{-1}$ (Fig. 4b, c, e and Supplementary Table 6). A subset of the most potent of these antibodies was also tested against authentic SARS-CoV-2 and these antibodies neutralized the virus with $\mathrm{IC}_{50}$ values of less than $5 \mathrm{ng} \mathrm{ml}^{-1}$ (Fig. $4 \mathrm{~d}$, e). Only two of the antibodies that cross-reacted with the RBD of SARS-CoV showed significant neutralizing activity against SARS-CoV pseudovirus (Extended Data Fig. 9d, e).

Potent neutralizing antibodies were found in individuals irrespective of their plasma $\mathrm{NT}_{50}$ values. For example, antibodies C121, C144 and $\mathrm{C135}$, which had $\mathrm{IC}_{50}$ values of $1.64,2.55$ and $2.98 \mathrm{ng} \mathrm{ml}^{-1}$ against authentic SARS-CoV-2, respectively, were obtained from individuals COV107, COV47 and COV72, for whom the plasma $\mathrm{NT}_{50}$ values were 297, 10,433 and 3,138, respectively (Figs. 2b, 4). Finally, antibodies with recurrent combinations of $I G H V$ and $I G L V$ genes were among the strongest neutralizing antibodies - for example, antibody $\mathrm{C} 002$ is composed of IGHV3-30/IGKV1-39 and shared by the two donors with the strongest plasma neutralizing activity (Figs. 3b, 4). We conclude that even individuals with modest plasma neutralizing activity have rare IgG memory B cells that produce potent SARS-CoV-2-neutralizing antibodies.

To determine whether human anti-SARS-CoV-2 monoclonal antibodies with neutralizing activity can bind to distinct domains on the RBD, we performed bilayer interferometry experiments in which a preformed antibody-RBD immune complex was exposed to a second monoclonal antibody. The antibodies tested comprised three groups, all of which differed in their binding properties from CR3022, an antibody 


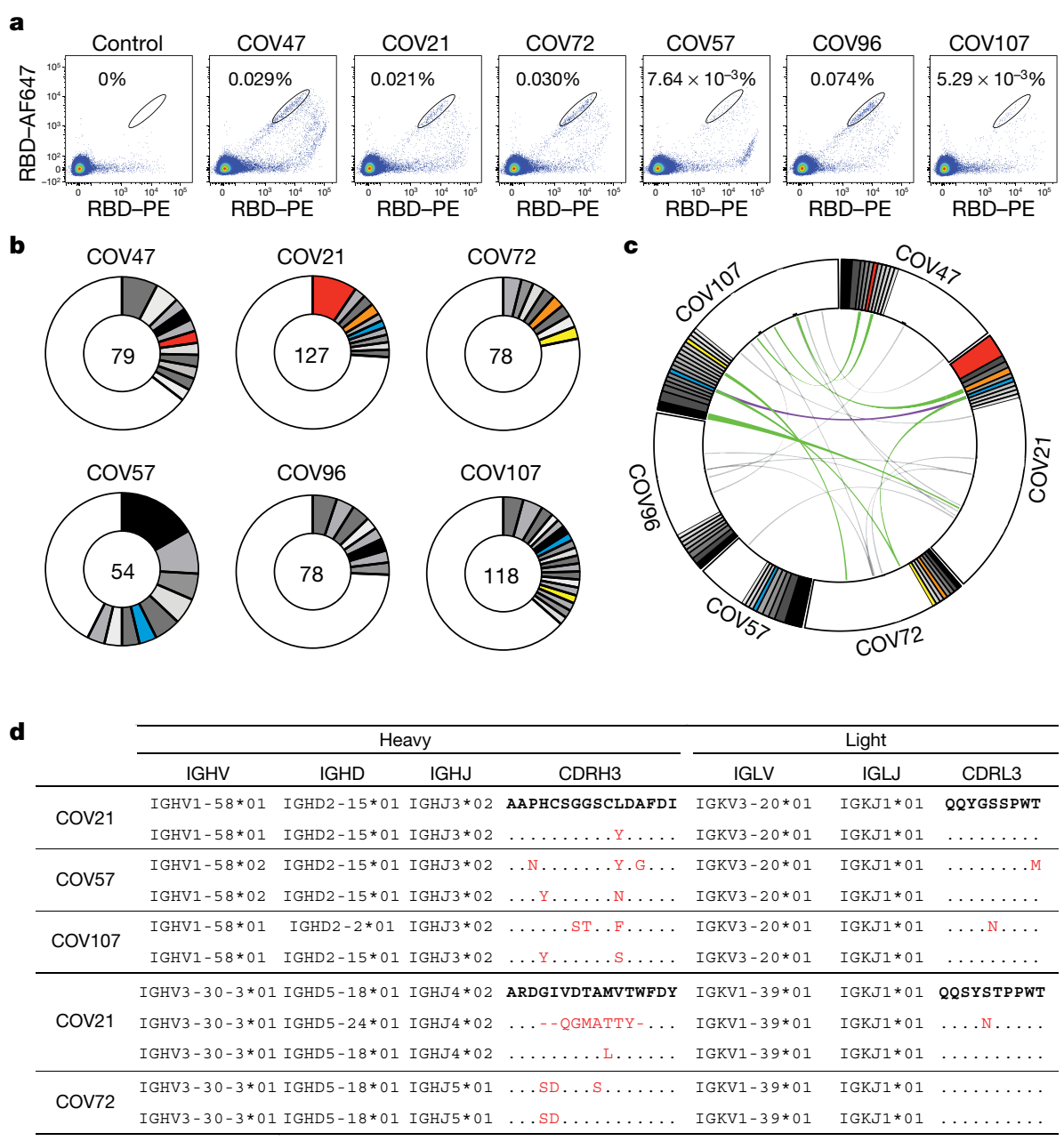

Fig. 3 | Anti-SARS-CoV-2 RBD antibodies. a, Representative flow cytometry plots showing dual AlexaFluor-647-RBD- and PE-RBD-binding B cells for one control and six study individuals (the gating strategy is shown in Extended Data Fig. 6). Percentages of antigen-specific $B$ cells are indicated. The control is a sample from a healthy individual obtained before COVID-19. b. The distribution of antibody sequences from six individuals. The number in the inner circle indicates the number of sequences analysed for the individual denoted above the circle. White indicates sequences isolated only once, and grey or coloured pie slices are proportional to the number of clonally related sequences. Red, blue, orange and yellow pie slices indicate clones that share the same $I G H V$ and
$I G L V$ genes.c, Sequences from all six individuals with clonal relationships depicted as in $\mathbf{b}$. Interconnecting lines indicate the relationship between antibodies that share $V$ and J gene segment sequences at both IGH and IGL. Purple, green and grey lines connect related clones, clones and singles, and singles to each other, respectively. d, Sample sequence alignment for antibodies originating from different individuals that display highly similar IGH V(D)J and IGL VJ sequences including CDR3s. Amino acid differences in CDR3s to the reference sequence (bold) are indicated in red, dashes indicate missing amino acids and dots represent identical amino acids. that neutralizes SARS-CoV and binds to-but does not neutralizeSARS-CoV- $2^{13,14}$. The antibodies of each of the three groups included: $\mathrm{C} 144$ and $\mathrm{C} 101$ in group $1 ; \mathrm{C} 121$ and $\mathrm{C} 009$ in group 2; $\mathrm{C} 135$ in group 3. All of these antibodies could bind to SARS-CoV-2 RBD that was previously immunocomplexed with CR3022. Groups 1 and 2 also bind to the RBD immunocomplexed with group 3 antibody. Groups 1 and 2 differ in that group 1 can bind to the RBD immunocomplexed with group 2 but not vice versa (Fig. $4 \mathrm{f}-\mathrm{n}$ ). We conclude that similar to SARS-CoV, there are multiple distinct neutralizing epitopes on the RBD of SARS-CoV-2.

To further define the binding characteristics of group-1 and group-2 antibodies, we imaged SARS-CoV-2 S-Fab complexes using negative-stain electron microscopy using C002 (group 1, an IGHV3-30/ IGKV1-39 antibody, which is clonally expanded in two donors), C119 and C121 (both in group 2) Fabs (Fig. $4 \mathrm{f}-\mathrm{r}$ and Extended Data Fig. 10). Consistent with the conformational flexibility of the RBD, two-dimensional class averages showed heterogeneity in both occupancy and orientations of bound Fabs for both groups (Fig. 4o-q). The low resolution of negative-stain electron-microscopy reconstructions precludes detailed binding interpretations; however, the results are consistent with Fabs from both groups being able to recognize 'up' and 'down' states of the RBD, as previously described for some antibodies targeting this epitope $\mathrm{e}^{15,16}$. The three-dimensional reconstructions are also consistent with competition measurements that indicate that group-1 and group-2 antibodies bind to a RBD epitope that is distinct from the epitope bound by antibody CR3022 (Fig. $4 \mathrm{f}-\mathrm{n}$ ) and with a single-particle cryo-electron microscopy structure of a C105-S comple ${ }^{17}$. In addition, the structures suggest that the antibodies bind to the RBD with different angles of approach; group-1 antibodies have an approach angle that is more similar to the approach angle of the SARS-CoV antibody $S 230^{18}$ (Fig. 4r).

Human monoclonal antibodies with neutralizing activity against pathogens ranging from viruses to parasites have been obtained from naturally infected individuals by single-cell antibody cloning. Several antibodies have been shown to be effective for the protection and treatment of model organisms and in early-phase clinical studies, but only one antiviral monoclonal antibody is currently in clinical use ${ }^{19}$. Antibodies are relatively expensive and more difficult to produce than small-molecule drugs. However, they differ from drugs in that they can engage the host immune system through their constant domains that 

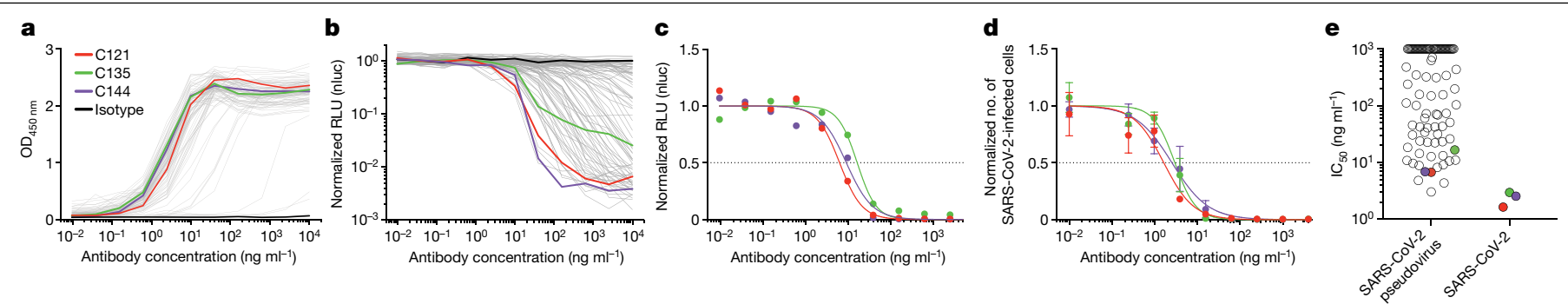

$\mathbf{f}$
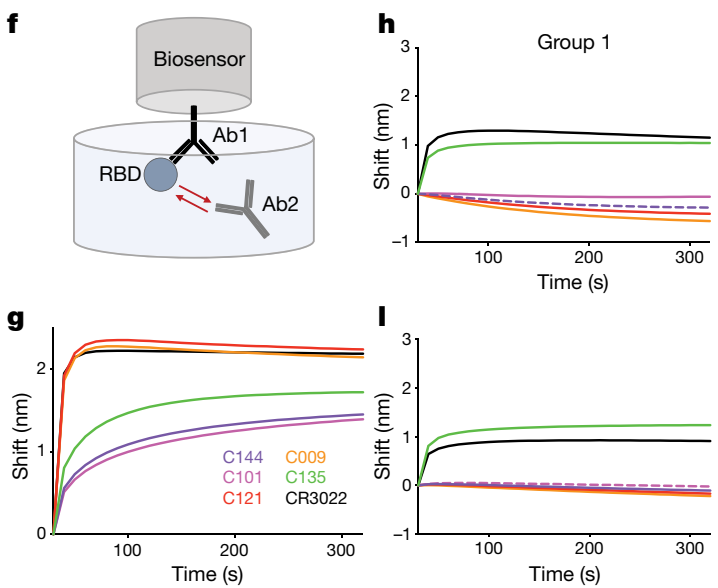
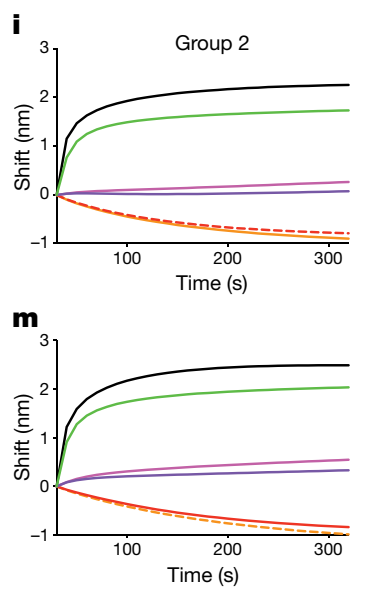
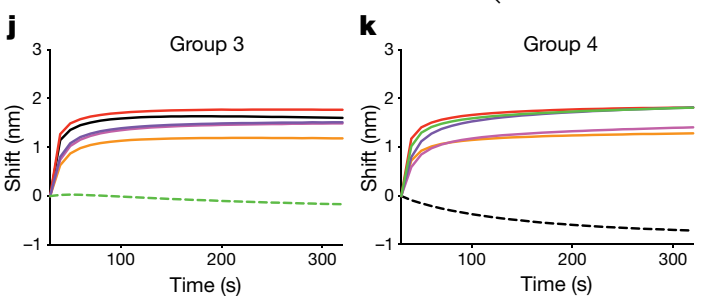

$\mathbf{0}$

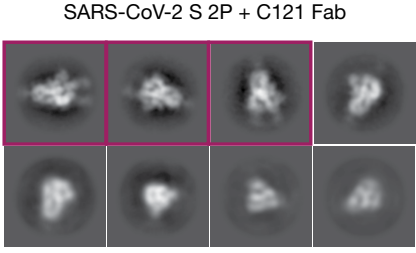

$\mathbf{p}$

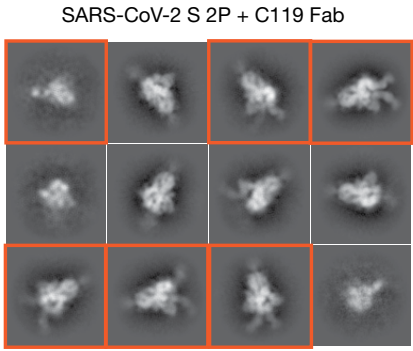

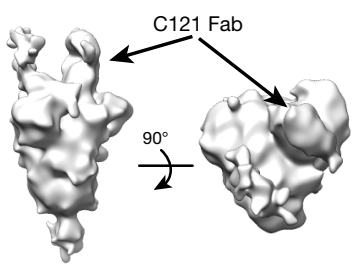

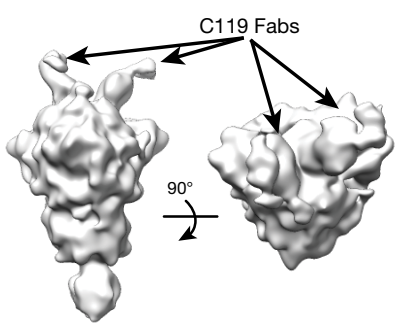

$\mathbf{q}$
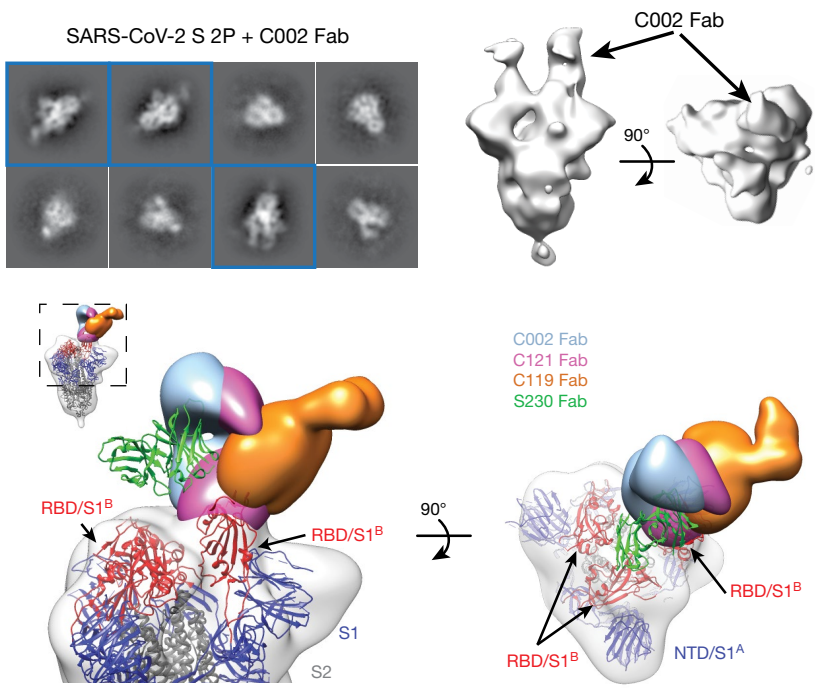

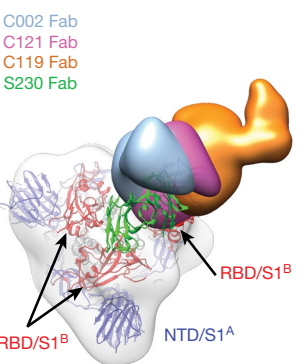

Fig. 4 | Anti-SARS-CoV-2 RBD antibody reactivity. a, Results of ELISAs measuring monoclonal antibody binding to RBD. $n=94$ samples and 1 isotype control. In all panels, C121, C135, C144 and isotype control are shown in red, green, purple and black, respectively.b. The normalized relative luminescence values for cell lysates of $293 \mathrm{~T}_{\mathrm{ACE} 2}$ cells $48 \mathrm{~h}$ after infection with SARS-CoV-2 pseudovirus in the presence of increasing concentrations of monoclonal antibodies. $n=89$ samples and 1 isotype control.c, SARS-CoV-2 pseudovirus neutralization assay. Normalized relative luminescence values were determined in the presence of a titration of monoclonal antibodies C121, C135 and C144.d, SARS-CoV-2 real virus neutralization assay. Normalized number of infected cells (determined by dividing the amount of infection per well by the average of control wells infected in the absence of antibodies) were determined in the presence of a titration of monoclonal antibodies $\mathrm{C} 121, \mathrm{C} 135$ and $\mathrm{C} 144$. a-d, Data are representative of two independent experiments. Data are the mean of duplicates $(\mathbf{b}, \mathbf{c})$ or mean \pm s.d. of triplicates (d).e, $\mathrm{IC}_{50}$ values for antibodies assayed in $\mathbf{b}$ and $\mathbf{d}$, the mean value of at least two experiments is shown. Samples with $\mathrm{IC}_{50}$ values above $1 \mu \mathrm{g} \mathrm{ml}^{-1}$ were plotted at $1 \mu \mathrm{g} \mathrm{ml}^{-1} . n=89$ (pseudovirus) and $n=3$ (virus). $\mathbf{f}$, Diagram of the biolayer

bind to Fc gamma receptors on host immune cells ${ }^{20}$. These interactions can enhance immunity and help to clear the pathogen or infected cells; however, they can also lead to disease enhancement during infections interferometry experiment. g, Binding of C144, C101, C121, C009, $\mathrm{C} 135$ and CR3022 $2^{13,14}$ to RBD. h-m, Second antibody (Ab2) binding to preformed first antibody (Ab1)-RBD complexes. Dotted line denotes when Ab1 and Ab2 are the same, and Ab2 is according to the colour-coding in $\mathbf{g} . \mathbf{h}, \mathbf{l}$, Group 1 antibodies were tested. $\mathrm{C} 144$ (h) and $\mathrm{C101}$ (I) were used as Ab1. i, $\mathbf{m}$, Group 2 antibodies were tested. $\mathrm{C121}(\mathbf{i})$ and $\mathrm{C009}(\mathbf{m})$ were used as Ab1.j, A group 3 antibody was tested. C135 was used as Ab1. k, Agroup 4 antibody was tested.CR3022 was used as Ab1.n, The shift in nanometres after Ab2 binding to the preformed Ab1-RBD complexes. Values are normalized through the subtraction of the autologous antibody control. Data are representative of two experiments. $\mathbf{o}-\mathbf{q}$, Representative two-dimensional class averages and three-dimensional reconstructed volumes for SARS-CoV-2S 2P trimers complexed with $\mathrm{C002}(\mathbf{q})$, $\mathrm{C} 119$ (p) and $\mathrm{C121}$ (o) Fabs. Two-dimensional class averages with observable Fab density are outlined.r, Overlay of S-Fab complexes with fully occupied C002 (blue), C121 (magenta) and C119 (orange) Fabs. The SARS-CoV-2S model from PDB 6VYB was fit into the density. The SARS-CoV monoclonal antibody S230 (PDB 6NB6) is shown as a reference (green ribbon).

with dengue virus ${ }^{21}$ and possibly coronavirus ${ }^{22}$. This problem has impeded the development of dengue virus vaccines, but would not interfere with the clinical use of potent neutralizing antibodies that 


\section{Article}

can be modified to prevent interactions with the Fc gamma receptor and that remain protective against viral pathogen $\mathrm{s}^{23}$.

Antibodies are essential elements of most vaccines and will probably be a crucial component of an effective vaccine against SARS-CoV-2 ${ }^{24-26}$. Recurrent antibodies have been observed in other infectious diseases and vaccine response ${ }^{11,27-30}$. The observation that plasma neutralizing activity is low in most convalescent individuals, but that recurrent anti-SARS-CoV-2 RBD antibodies with potent neutralizing activity can be found in individuals with moderate plasma neutralizing activity suggests that humans are intrinsically capable of generating anti-RBD antibodies that potently neutralize SARS-CoV-2. Thus, vaccines that selectively and efficiently induce antibodies that target the RBD of SARS-CoV-2 may be especially effective.

\section{Online content}

Any methods, additional references, Nature Research reporting summaries, source data, extended data, supplementary information, acknowledgements, peer review information; details of author contributions and competing interests; and statements of data and code availability are available at https://doi.org/10.1038/s41586-020-2456-9.

1. Graham, R. L., Donaldson, E. F. \& Baric, R. S. A decade after SARS: strategies for controlling emerging coronaviruses. Nat. Rev. Microbiol. 11, 836-848 (2013).

2. Gralinski, L. E. \& Baric, R. S. Molecular pathology of emerging coronavirus infections. J. Pathol. 235, 185-195 (2015).

3. Hoffmann, M. et al SARS-CoV-2 cell entry depends on ACE2 and TMPRSS2 and is blocked by a clinically proven protease inhibitor. Cell 181, 271-280 (2020).

4. Walls, A. C. et al. Structure, function, and antigenicity of the SARS-CoV-2 spike glycoprotein. Cell 181, 281-292 (2020).

5. Jiang, S., Hillyer, C. \& Du, L. Neutralizing antibodies against SARS-CoV-2 and other human coronaviruses. Trends Immunol. 41, 355-359 (2020).

6. Amanat, F. et al. A serological assay to detect SARS-CoV-2 seroconversion in humans. Nat. Med. https://doi.org/10.1038/s41591-020-0913-5 (2020).

7. Grifoni, A. et al. Targets of T cell responses to SARS-CoV-2 coronavirus in humans with COVID-19 disease and unexposed individuals. Cell 181, 1489-1501 (2020).

8. Scheid, J. F. et al. Broad diversity of neutralizing antibodies isolated from memory B cells in HIV-infected individuals. Nature 458, 636-640 (2009).

9. Tiller, T. et al. Autoreactivity in human IgG ${ }^{+}$memory B cells. Immunity 26, 205-213 (2007)

10. Murugan, R. et al. Clonal selection drives protective memory $B$ cell responses in controlled human malaria infection. Sci. Immunol. 3, eaap8029 (2018).
11. Wang, Q. et al. A combination of human broadly neutralizing antibodies against hepatitis $B$ virus $\mathrm{HBsAg}$ with distinct epitopes suppresses escape mutations. Cell Host Microbe https://doi.org/10.1016/j.chom.2020.05.010 (2020).

12. Briney, B., Inderbitzin, A., Joyce, C. \& Burton, D. R. Commonality despite exceptional diversity in the baseline human antibody repertoire. Nature 566, 393-397 (2019).

13. ter Meulen, J. et al. Human monoclonal antibody combination against SARS coronavirus: synergy and coverage of escape mutants. PLoS Med. 3, e237 (2006).

14. Yuan, M. et al. A highly conserved cryptic epitope in the receptor binding domains of SARS-CoV-2 and SARS-CoV. Science 368, 630-633 (2020).

15. Walls, A. C. et al. Unexpected receptor functional mimicry elucidates activation of coronavirus fusion. Cell 176, 1026-1039 (2019)

16. Pinto, D. et al. Cross-neutralization of SARS-CoV-2 by a human monoclonal SARS-CoV antibody. Nature 583, 290-295 (2020).

17. Barnes, C. O. et al. Structures of human antibodies bound to SARS-CoV-2 spike reveal common epitopes and recurrent features of antibodies. Cell https://doi.org/10.1016/j. cell.2020.06.025 (2020).

18. Zhu, Z. et al. Potent cross-reactive neutralization of SARS coronavirus isolates by human monoclonal antibodies. Proc. Natl Acad. Sci. USA 104, 12123-12128 (2007)

19. Salazar, G., Zhang, N., Fu, T. M. \& An, Z. Antibody therapies for the prevention and treatment of viral infections. NPJ Vaccines 2, 19 (2017)

20. Bournazos, S. \& Ravetch, J. V. Anti-retroviral antibody FcyR-mediated effector functions. Immunol. Rev. 275, 285-295 (2017)

21. Feinberg, M. B. \& Ahmed, R. Advancing dengue vaccine development. Science $\mathbf{3 5 8}$, 865-866 (2017)

22. Iwasaki, A. \& Yang, Y. The potential danger of suboptimal antibody responses in COVID-19. Nat. Rev. Immunol. 20, 339-341 (2020).

23. Van Rompay, K. K. A. et al. A combination of two human monoclonal antibodies limits fetal damage by Zika virus in macaques. Proc. Natl Acad. Sci. USA 117, 7981-7989 (2020)

24. Plotkin, S. A. Correlates of protection induced by vaccination. Clin. Vaccine Immunol. 17, 1055-1065 (2010).

25. $\mathrm{Yu}$, J. et al. DNA vaccine protection against SARS-CoV-2 in rhesus macaques. Science eabc6284 (2020).

26. Chandrashekar, A. et al. SARS-CoV-2 infection protects against rechallenge in rhesus macaques. Science eabc4776 (2020)

27. Scheid, J. F. et al. Sequence and structural convergence of broad and potent HIV antibodies that mimic CD4 binding. Science 333, 1633-1637 (2011)

28. Robbiani, D. F. et al. Recurrent potent human neutralizing antibodies to Zika virus in Brazil and Mexico. Cell 169, 597-609 (2017).

29. Ehrhardt, S. A. et al. Polyclonal and convergent antibody response to Ebola virus vaccine rVSV-ZEBOV. Nat. Med. 25, 1589-1600 (2019).

30. Pappas, L. et al. Rapid development of broadly influenza neutralizing antibodies through redundant mutations. Nature 516, 418-422 (2014).

Publisher's note Springer Nature remains neutral with regard to jurisdictional claims in published maps and institutional affiliations.

c The Author(s), under exclusive licence to Springer Nature Limited 2020 


\section{Methods}

\section{Data reporting}

No statistical methods were used to predetermine sample size. The experiments were not randomized and the investigators were not blinded to allocation during experiments and outcome assessment.

\section{Study participants}

Study participants were recruited at the Rockefeller University Hospital in New York from 1 April to 8 May 2020. Eligible participants were adults aged $18-76$ years who were either diagnosed with a SARS-CoV-2 infection by RT-PCR and were free of symptoms of COVID-19 for at least 14 days (cases), or who were close contacts (for example, household members, co-workers or members of same religious community) of someone who had been diagnosed with a SARS-CoV-2 infection by RT-PCR and were free of symptoms suggestive of COVID-19 for at least 14 days (contacts). Exclusion criteria included the presence of symptoms suggestive of an active SARS-CoV-2 infection, or haemoglobin levels of $<12 \mathrm{~g} / \mathrm{dl}$ for men and $<11 \mathrm{~g} / \mathrm{dl}$ for women.

Most study participants were residents of the Greater New York City tristate region and were enrolled sequentially according to eligibility criteria. Participants were first interviewed by phone to collect information on their clinical presentation, and subsequently presented to the Rockefeller University Hospital for the collection of a single blood sample. Participants were asked to rate the highest severity of their symptoms on a numeric rating scale ranging from 0 to 10 . The score was adapted from the pain scale chart, in which 0 was the lack of symptoms, 4 was distressing symptoms (for example, fatigue, myalgia, fever, cough or shortness of breath) that interfered with daily living activities, 7 was disabling symptoms that prevented the performance of daily living activities, and 10 was unimaginable/unspeakable discomfort (in this case, distress owing to shortness of breath). All participants provided written informed consent before participation in the study and the study was conducted in accordance with Good Clinical Practice and clinical data collection and management were carried out using the software iRIS by iMedRIS. The study was performed in compliance with all relevant ethical regulations and the protocol for studies with human participants was approved by the Institutional Review Board of the Rockefeller University.

\section{Blood samples processing and storage}

Peripheral blood mononuclear cells were obtained by gradient centrifugation and stored in liquid nitrogen in the presence of fetal calf serum (FCS) and DMSO. Heparinized plasma and serum samples were aliquoted and stored at $-20^{\circ} \mathrm{C}$ or less. Before experiments, aliquots of plasma samples were heat-inactivated $\left(56^{\circ} \mathrm{C}\right.$ for $\left.1 \mathrm{~h}\right)$ and then stored at $4{ }^{\circ} \mathrm{C}$.

\section{Cloning, expression and purification of recombinant coronavirus proteins}

Codon-optimized nucleotide sequences encoding the SARS-CoV-2 S ectodomain (residues 16-1206) and RBD (residues 331-524) were synthesized and subcloned into the mammalian expression pTwist-CMV BetaGlobin vector by Twist Bioscience Technologies based on an early SARS-CoV-2 sequence isolate (GenBank MN985325.1). The SARS-CoV-2 RBD construct included an N-terminal human IL-2 signal peptide and dual C-terminal tags ((GGGGS) ${ }_{2}$-HHHHHHHH (octa-histidine) and GLNDIFEAQKIEWHE (AviTag)). In addition, the corresponding $\mathrm{S1}^{\mathrm{B}}$ or RBDs for SARS-CoV (residues 318-510; GenBank AAP13441.1), MERS-CoV (residues 367-588; GenBankJX869059.2), HCoV-NL63 (residues 481-614; GenBank AAS58177.1), HCoV-OC43 (residues 324-632; GenBank AAT84362.1) and HCoV-229E (residues 286-434; GenBank AAK32191.1) were synthesized with the same $\mathrm{N}$ - and C-terminal extensions as the SARS-CoV-2 RBD construct and subcloned into the mammalian expression pTwist-CMV BetaGlobin vector (Twist Bioscience
Technologies). The SARS-CoV-2 S ectodomain was modified as previously described ${ }^{4}$. In brief, the $S$ ectodomain construct included an $\mathrm{N}$-terminal mu-phosphatase signal peptide, 2P stabilizing mutations (K986P and V987P), mutations to remove the S1/S2 furin cleavage site $\left({ }_{682} \mathrm{RRAR}_{685}\right.$ to GSAS), a C-terminal extension (IKGSG-RENLYFQG (TEV protease site), GGGSG-YIPEAPRDGQAYVRKDGEWVLLSTFL (foldon trimerization motif), G-HHHHHHHH (octa-histidine tag) and GLNDIFEAQKIEWHE (AviTag)). The SARS-CoV-2 S 2P ectodomain and RBD constructs were produced by transient transfection of $500 \mathrm{ml}$ of Expi293F cells (Thermo Fisher) and purified from clarified transfected cell supernatants 4 days after transfection using $\mathrm{Ni}^{2+}$-NTA affinity chromatography (GE Life Sciences). Affinity-purified proteins were concentrated and further purified by size-exclusion chromatography using a Superdex200 16/60 column (GE Life Sciences) running in $1 \times$ TBS (20 mM Tris- $\mathrm{HCl} \mathrm{pH} \mathrm{8.0,150} \mathrm{mM} \mathrm{NaCl}$ and $0.02 \% \mathrm{NaN}_{3}$ ). Peak fractions were analysed by SDS-PAGE, and fractions corresponding to soluble $S$ $2 \mathrm{P}$ trimers or monomeric RBD proteins were pooled and stored at $4{ }^{\circ} \mathrm{C}$.

\section{ELISAs}

Validated ELISAs $s^{6,7}$ to evaluate antibodies binding to SARS-CoV-2 RBD and trimeric spike proteins, and to SARS-CoV RBD, were performed by coating of high-binding 96-half-well plates (Corning 3690 ) with $50 \mu \mathrm{l}$ per well of a $1 \mu \mathrm{g} / \mathrm{ml}$ protein solution in PBS overnight at $4{ }^{\circ} \mathrm{C}$. Plates were washed 6 times with washing buffer $(1 \times$ PBS with $0.05 \%$ Tween-20 (Sigma-Aldrich)) and incubated with $170 \mu$ l per well blocking buffer ( $1 \times$ PBS with $2 \%$ BSA and $0.05 \%$ Tween-20 (Sigma)) for $1 \mathrm{~h}$ at room temperature. Immediately after blocking, monoclonal antibodies or plasma samples were added in PBS and incubated for $1 \mathrm{~h}$ at room temperature. Plasma samples were assayed at a 1:200 starting dilution and 7 additional threefold serial dilutions. Monoclonal antibodies were tested at $10 \mu \mathrm{g} / \mathrm{ml}$ starting concentration and 10 additional fourfold serial dilutions. Plates were washed 6 times with washing buffer and then incubated with anti-human IgG or IgM secondary antibody conjugated to horseradish peroxidase (HRP) (Jackson Immuno Research 109-036-088 and 109-035-129) in blocking buffer at a 1:5,000 dilution. Plates were developed by addition of the HRP substrate, TMB (ThermoFisher) for $10 \mathrm{~min}$, then the developing reaction was stopped by adding $50 \mu \mathrm{I} 1 \mathrm{M} \mathrm{H}_{2} \mathrm{SO}_{4}$ and absorbance was measured at $450 \mathrm{~nm}$ with an ELISA microplate reader (FluoStar Omega, BMG Labtech) with Omega and Omega MARS software for analysis. For plasma samples, a positive control (plasma from patient COV21, diluted 200-fold in PBS) and negative control (historical plasma samples) samples were added in duplicate to every assay plate for validation. The average of its signal was used for normalization of all of the other values on the same plate with Excel software before calculating the area under the curve using Prism 8 (GraphPad). For monoclonal antibodies, the $\mathrm{EC}_{50}$ was determined using four-parameter nonlinear regression (GraphPad Prism).

\section{T $_{\text {ACE2 }}$ cells}

For constitutive expression of ACE2 in 293T cells, a cDNA encoding ACE2, carrying two inactivating mutations in the catalytic site $(\mathrm{H} 374 \mathrm{~N}$ and $\mathrm{H} 378 \mathrm{~N}$ ), was inserted into CSIB 3 ' to the SFFV promoter ${ }^{31} .293 \mathrm{~T}_{\mathrm{ACE} 2}$ cells were generated by transduction with CSIB-based virus followed by selection with $5 \mu \mathrm{g} / \mathrm{ml}$ blasticidin.

\section{SARS-CoV-2 and SARS-CoV pseudotyped reporter viruses}

A plasmid expressing a C-terminally truncated SARS-CoV-2 S protein (pSARS-CoV2-S $S_{\text {trunc }}$ ) was generated by insertion of a human codon-optimized cDNA encoding SARS-CoV-2S lacking the C-terminal 19 codons (Geneart) into pCR3.1. The S open-reading frame was taken from 'Wuhan seafood market pneumonia virus isolate Wuhan-Hu-1' (GenBank: NC_045512). For expression of the full-length SARS-CoV S protein, 'human SARS coronavirus spike glycoprotein gene ORF cDNA clone expression plasmid (codon optimized)' (here referred to as pSARS-CoV-S) was obtained from SinoBiological (VG40150-G-N). 
An env-inactivated HIV-1 reporter construct (pNL4-3 $\Delta$ Env-nanoluc) was generated from pNL4- $3^{32}$ by introducing a 940-bp deletion $3^{\prime}$ in the $v p u$ stop codon, resulting in a frameshift in env. The human codon-optimized nanoluc Luciferase reporter gene (Nluc, Promega) was inserted in place of nucleotides 1-100 of the nef gene. To generate pseudotyped viral stocks, 293T cells were transfected with pNL4-3 $\Delta$ Env-nanoluc and pSARS-CoV2-S trunc $_{\text {or }}$ pSARS-CoV-S using polyethylenimine. Co-transfection of pNL4-3 $\Delta$ Env-nanoluc and $\mathrm{S}$-expression plasmids leads to production of HIV-1-based virions that carried either the SARS-CoV-2 or SARS-CoV S protein on the surface. After transfection for $8 \mathrm{~h}$, cells were washed twice with PBS and fresh medium was added. Supernatants containing virions were collected $48 \mathrm{~h}$ after transfection, filtered and stored at $-80^{\circ} \mathrm{C}$. Infectivity of virions was determined by titration on $293 \mathrm{~T}_{\mathrm{ACE} 2}$ cells. Further details are described elsewhere ${ }^{33}$.

\section{Pseudotyped virus neutralization assay}

Fivefold serially diluted plasma from COVID-19-convalescent individuals and healthy donors or fourfold serially diluted monoclonal antibodies were incubated with the SARS-CoV-2 or SARS-CoV pseudotyped virus for $1 \mathrm{~h}$ at $37^{\circ} \mathrm{C}$. The mixture was subsequently incubated with $293 \mathrm{~T}_{\mathrm{ACE} 2}$ cells for $48 \mathrm{~h}$ after which cells were washed twice with PBS and lysed with Luciferase Cell Culture Lysis $5 \times$ reagent (Promega). Nanoluc Luciferase activity in lysates was measured using the Nano-Glo Luciferase Assay System (Promega) with Modulus II Microplate Reader User interface (TURNER BioSystems). The obtained relative luminescence units were normalized to those derived from cells infected with SARS-CoV-2 or SARS-CoV pseudotyped virus in the absence of plasma or monoclonal antibodies. The half-maximal inhibitory concentration for plasma $\left(\mathrm{NT}_{50}\right)$ or monoclonal antibodies $\left(\mathrm{IC}_{50}\right)$ was determined using four-parameter nonlinear regression (GraphPad Prism).

\section{Cell lines, virus and virus titration}

VeroE6 kidney epithelial cells (Chlorocebus sabaeus; ATCC) and Huh7.5 hepatoma cells (Homo sapiens; C.M.R.) were cultured in Dulbecco's modified Eagle medium (DMEM) supplemented with $1 \%$ nonessential amino acids and $10 \% \mathrm{FCS}$ at $37^{\circ} \mathrm{C}$ and $5 \% \mathrm{CO}_{2}$. All cell lines have been tested negative for contamination with mycoplasma and were obtained from the ATCC (with the exception for Huh-7.5). SARS-CoV-2, strain USA-WA1/2020, was obtained from BEI Resources and amplified in VeroE 6 cells at $33^{\circ} \mathrm{C}$. Viral titres were measured on Huh- 7.5 cells by standard plaque assay. In brief, $500 \mu \mathrm{l}$ of serial tenfold virus dilutions in Opti-MEM were used to infect 400,000 cells seeded the previous day in a 6-well plate format. After $90 \mathrm{~min}$ adsorption, the virus inoculum was removed, and cells were overlayed with DMEM containing $10 \%$ FCS with $1.2 \%$ microcrystalline cellulose (Avicel). Cells were incubated for 5 days at $33^{\circ} \mathrm{C}$, followed by fixation with $3.5 \%$ formaldehyde and crystal violet staining for plaque enumeration. All experiments were performed in a biosafety level 3 laboratory.

Microscopy-based neutralization assay of authentic SARS-CoV-2 The day before infection, VeroE6 cells were seeded at 12,500 cells/well into 96-well plates. Antibodies were serially diluted in BA-1, mixed with a constant amount of SARS-CoV-2 (grown in VeroE6) and incubated for $60 \mathrm{~min}$ at $37^{\circ} \mathrm{C}$. The antibody-virus mix was then directly applied to VeroE6 cells (MOI of $\sim 0.1 \mathrm{PFU} /$ cell). Cells were fixed $18 \mathrm{~h}$ after infection by adding an equal volume of $7 \%$ formaldehyde to the wells, followed by permeabilization with $0.1 \%$ Triton X-100 for $10 \mathrm{~min}$. After extensive washing, cells were incubated for $1 \mathrm{~h}$ at room temperature with blocking solution of $5 \%$ goat serum in PBS (005-000-121; Jackson ImmunoResearch). A rabbit polyclonal anti-SARS-CoV-2 nucleocapsid antibody (GTX135357; GeneTex) was added to the cells at 1:500 dilution in blocking solution and incubated at $4{ }^{\circ} \mathrm{C}$ overnight. A goat anti-rabbit AlexaFluor 594 (A-11012; Life Technologies) at a dilution of 1:2,000 was used as a secondary antibody. Nuclei were stained with
Hoechst 33342 (62249; Thermo Scientific) at a 1:1,000 dilution. Images were acquired with a fluorescence microscope and analysed using ImageXpress Micro XLS and MetaXpress software (Molecular Devices). All statistical analyses were done using Prism 8 software (GraphPad).

\section{Biotinylation of viral protein for use in flow cytometry}

Purified and Avi-tagged SARS-CoV-2 RBD was biotinylated using the Biotin-Protein Ligase-BIRA kit according to manufacturer's instructions (Avidity). Ovalbumin (Sigma, A5503-1G) was biotinylated using the EZ-Link Sulfo-NHS-LC-Biotinylation kit according to the manufacturer's instructions (Thermo Scientific). Biotinylated ovalbumin was conjugated to streptavidin-BV711 (BD biosciences, 563262) and RBD to streptavidin-PE (BD Biosciences, 554061) and streptavidin-AF647 (Biolegend, 405237) ${ }^{34}$.

\section{Single-cell sorting by flow cytometry}

Peripheral blood mononuclear cells were enriched for B cells by negative selection using a pan-B-cell isolation kit according to the manufacturer's instructions (Miltenyi Biotec, 130-101-638). The enriched $B$ cells were incubated in FACS buffer ( $1 \times$ PBS, $2 \%$ FCS, 1 mM EDTA) with the following anti-human antibodies (all at 1:200 dilution): anti-CD20-PECy7 (BD Biosciences, 335793), anti-CD3-APC-eFluro 780 (Invitrogen, 47-0037-41), anti-CD8-APC-eFluor 780 (Invitrogen, 47-0086-42), anti-CD16-APC-eFluor 780 (Invitrogen, 47-0168-41), anti-CD14-APC-eFluor 780 (Invitrogen, 47-0149-42), as well as Zombie NIR (BioLegend, 423105) and fluorophore-labelled RBD and ovalbumin (Ova) for 30 min on ice ${ }^{34}$. Single CD ${ }^{-} \mathrm{CD}^{-} \mathrm{CD} 14^{-} \mathrm{CD} 16^{-} \mathrm{CD} 20^{+} \mathrm{Ova}^{-} \mathrm{RBD}$ $\mathrm{PE}^{+} \mathrm{RBD}-\mathrm{AF} 647^{+} \mathrm{B}$ cells were sorted into individual wells of 96 -well plates containing $4 \mu \mathrm{l}$ of lysis buffer ( $0.5 \times$ PBS, $10 \mathrm{mM} \mathrm{DTT,} \mathrm{3,000} \mathrm{units} / \mathrm{ml}$ RNasin Ribonuclease Inhibitors (Promega, N2615) per well using a FACS Aria III and FACSDiva software (Becton Dickinson) for acquisition and FlowJo for analysis. The sorted cells were frozen on dry ice, and then stored at $-80^{\circ} \mathrm{C}$ or immediately used for subsequent RNA reverse transcription. Although cells were not stained for IgG expression, they are memory B cells based on the fact that they are $\mathrm{CD} 2 \mathrm{O}^{+}$(a marker that is absent in plasmablasts) and they express IgG (as antibodies were amplified from these cells using IgG-specific primers).

\section{Antibody sequencing, cloning and expression}

Antibodies were identified and sequenced as described previously $^{28,35,36}$. In brief, RNA from single cells was reverse-transcribed (SuperScript III Reverse Transcriptase, Invitrogen, 18080-044) and the cDNA stored at $-20^{\circ} \mathrm{C}$ or used for subsequent amplification of the variable IGH, IGL and IGK genes by nested PCR and Sanger sequencing $^{35}$. Anti-Zika virus monoclonal antibody $Z 021^{28}$ was used as isotype control. Sequence analysis was performed using MacVector. Amplicons from the first PCR reaction were used as templates for sequenceand ligation-independent cloning into antibody expression vectors. Recombinant monoclonal antibodies and Fabs were produced and purified as previously described ${ }^{37,38}$.

\section{Biolayer interferometry}

Biolayer interferometry assays were performed on the Octet Red instrument (ForteBio) at $30^{\circ} \mathrm{C}$ with shaking at 1,000 r.p.m. Epitope-binding assays were performed with protein A biosensor (ForteBio 18-5010), following the manufacturer's protocol 'classical sandwich assay'. (1) Sensor check: sensors immersed 30 s in buffer alone (buffer ForteBio 18-1105). (2) Capture first antibody: sensors immersed 10 min with Ab1 at $40 \mu \mathrm{g} / \mathrm{ml}$. (3) Baseline: sensors immersed $30 \mathrm{~s}$ in buffer alone. (4) Blocking: sensors immersed $5 \mathrm{~min}$ with IgG isotype control at $50 \mu \mathrm{g} / \mathrm{ml}$. (6) Antigen association: sensors immersed 5 min with RBD at $100 \mu \mathrm{g} / \mathrm{ml}$. (7) Baseline: sensors immersed $30 \mathrm{~s}$ in buffer alone. (8) Association $\mathrm{Ab} 2$ : sensors immersed $5 \mathrm{~min}$ with $\mathrm{Ab} 2$ at $40 \mu \mathrm{g} / \mathrm{ml}$. Curve fitting was performed using the Fortebio Octet Data analysis software (ForteBio). 


\section{Computational analyses of antibody sequences}

Antibody sequences were trimmed based on quality and annotated using Igblastn v.1.14.0 ${ }^{39}$ with IMGT domain delineation system. Annotation was performed systematically using Change-O toolkit v.0.4.5 ${ }^{40}$. Heavy and light chains derived from the same cell were paired, and clonotypes were assigned based on their $\mathrm{V}$ and $\mathrm{J}$ genes using in-house $\mathrm{R}$ and Perl scripts (Fig. 3b, c). All scripts and the data used to process antibody sequences are publicly available on GitHub (https://github. com/stratust/igpipeline).

The frequency distributions of human $\mathrm{V}$ genes in anti-SARS-CoV-2 antibodies from this study were compared to Sequence Read Archive accession SRP010970 ${ }^{41}$. The V(D)J assignments were done using IMGT/High V-Quest and the frequencies of heavy and light chain V genes were calculated for 14 and 13 individuals, respectively, using sequences with unique CDR3s. The two-tailed $t$-test with unequal variances was used to determine statistical significance (Extended Data Fig. 7).

Nucleotide somatic hypermutation and CDR3 length were determined using in-house R and Perl scripts. For somatic hypermutations, IGHV and IGLV nucleotide sequences were aligned against their closest germlines using Igblastn and the number of differences were considered nucleotide mutations. The average mutations for $\mathrm{V}$ genes was calculated by dividing the sum of all nucleotide mutations across all patients by the number of sequences used for the analysis. To calculate the GRAVY scores of hydrophobicity ${ }^{42}$ we used Guy H.R. Hydrophobicity scale based on free energy of transfer $(\mathrm{kcal} / \mathrm{mole})^{43}$ implemented by the R package Peptides (the Comprehensive R Archive Network repository; https://journal.r-project.org/archive/2015/RJ-2015-001/ RJ-2015-001.pdf). We used 533 heavy chain CDR3 amino acid sequences from this study (sequence COV047_P4_IgG_51-P1369 lacks CDR3 amino acid sequence) and 22,654,256 IGH CDR3 sequences from the public database of memory B cell receptor sequences ${ }^{44}$. The Shapiro-Wilk test was used to determine whether the GRAVY scores are normally distributed. The GRAVY scores from all 533 IGH CDR3 amino acid sequences from this study were used to perform the test and 5,000 GRAVY scores of the sequences from the public database were randomly selected. The Shapiro-Wilk $P$ values were $6.896 \times 10^{-3}$ and $2.217 \times 10^{-6}$ for sequences from this study and the public database, respectively, indicating that the data were not normally distributed. Therefore, we used the Wilcoxon nonparametric test to compare the samples, which indicated a difference in hydrophobicity distribution $\left(P=5 \times 10^{-6}\right)$ (Extended Data Fig. 8).

\section{Negative-stain electron-microscopy data collection and processing}

Purified Fabs (C002, C119 and C121) were complexed with SARS-CoV-2 S trimer at a twofold molar excess for $1 \mathrm{~min}$ and diluted to $40 \mu \mathrm{g} / \mathrm{ml}$ in TBS immediately before adding $3 \mu \mathrm{l}$ to a freshly glow-discharged ultrathin, 400-mesh carbon-coated copper grid (Ted Pella). Samples were blotted after a 1-min incubation period and stained with $1 \%$ uranyl formate for an additional minute before imaging. Micrographs were recorded on a Thermo Fisher Talos Arctica transmission electron microscope operating at $200 \mathrm{keV}$ using a K3 direct electron detector (Gatan) and SerialEM automated image-acquisition software ${ }^{45}$. Images were acquired at a nominal magnification of $28,000 \times(1.44 \AA /$ pixel size) and a defocus range of -1.5 to $-2.0 \mu \mathrm{m}$. Images were processed in cryoSPARC, and reference-free particle picking was completed using a Gaussian blob picker ${ }^{46}$. Reference-free two-dimensional class averages and ab initio volumes were generated in cryoSPARC, and subsequently three-dimensionally classified to identify classes of S-Fab complexes, that were then homogenously refined. Figures were prepared using UCSF Chimera ${ }^{47}$. The resolutions of the final single-particle reconstructions were about 17-20 A calculated using a gold-standard FSC ( 0.143 cut-off) and about $24-28$ Å using a 0.5 cut-off.

\section{Reporting summary}

Further information on research design is available in the Nature Research Reporting Summary linked to this paper.

\section{Data availability}

Data are provided in Supplementary Tables 1,3-6. The raw sequencing data associated with Fig. 3 has been deposited at Github (https://github. com/stratust/igpipeline). This study also uses data from 'A public database of memory and naive B-cell receptor sequences' (https://doi. org/10.5061/dryad.35ks2), from PDB (6VYB and 6NB6) and from NCBI Sequence Read Archive (SRP010970).

\section{Code availability}

Computer code to process the antibody sequences is available at GitHub (https://github.com/stratust/igpipeline).

31. Kane, M. et al. Identification of interferon-stimulated genes with antiretroviral activity. Cell Host Microbe 20, 392-405 (2016).

32. Adachi, A. et al. Production of acquired immunodeficiency syndrome-associated retrovirus in human and nonhuman cells transfected with an infectious molecular clone. J. Virol. 59, 284-291 (1986).

33. Schmidt, F. et al. Measuring SARS-CoV-2 neutralizing antibody activity using pseudotyped and chimeric viruses. J. Exp. Med. https://doi.org/10.1084/jem.20201181 (2020).

34. Wang, Z. et al. Isolation of single HIV-1 envelope specific B cells and antibody cloning from immunized rhesus macaques. J. Immunol. Methods 478, 112734 (2020).

35. Tiller, $\mathrm{T}$. et al. Efficient generation of monoclonal antibodies from single human B cells by single cell RT-PCR and expression vector cloning. J. Immunol. Methods 329, 112-124 (2008).

36. von Boehmer, L. et al. Sequencing and cloning of antigen-specific antibodies from mouse memory B cells. Nat. Protocols 11, 1908-1923 (2016).

37. Klein, F. et al. Enhanced HIV-1 immunotherapy by commonly arising antibodies that target virus escape variants. J. Exp. Med. 211, 2361-2372 (2014).

38. Schoofs, T. et al. Broad and potent neutralizing antibodies recognize the silent face of the HIV envelope. Immunity 50, 1513-1529 (2019).

39. Ye, J., Ma, N., Madden, T. L. \& Ostell, J. M. IgBLAST: an immunoglobulin variable domain sequence analysis tool. Nucleic Acids Res. 41, W34-W40 (2013).

40. Gupta, N. T. et al. Change-O: a toolkit for analyzing large-scale B cell immunoglobulin repertoire sequencing data. Bioinformatics 31, 3356-3358 (2015).

41. Rubelt, F. et al. Onset of immune senescence defined by unbiased pyrosequencing of human immunoglobulin mRNA repertoires. PLoS ONE 7, e49774 (2012).

42. Kyte, J. \& Doolittle, R. F. A simple method for displaying the hydropathic character of a protein. J. Mol. Biol. 157, 105-132 (1982).

43. Guy, H. R. Amino acid side-chain partition energies and distribution of residues in soluble proteins. Biophys. J. 47, 61-70 (1985).

44. DeWitt, W. S. et al. A public database of memory and naive B-cell receptor sequences. PLOS ONE 11, e0160853 (2016).

45. Mastronarde, D. N. Automated electron microscope tomography using robust prediction of specimen movements. J. Struct. Biol. 152, 36-51 (2005).

46. Punjani, A., Rubinstein, J. L., Fleet, D. J. \& Brubaker, M. A. cryoSPARC: algorithms for rapid unsupervised cryo-EM structure determination. Nat. Methods 14, 290-296 (2017).

47. Goddard, T. D., Huang, C. C. \& Ferrin, T. E. Visualizing density maps with UCSF Chimera. J. Struct. Biol. 157, 281-287 (2007)

Acknowledgements We thank all study participants who devoted time to our research; B. Coller and S. Schlesinger, the Rockefeller University Hospital Clinical Research Support Office and nursing staff; J. L. DeRisi for facilitating interactions with the Chan Zuckerberg Biohub; all members of the M.C.N. laboratory for discussions; A. Escolano, G. Breton and B. Reis; M. Jankovic for laboratory support; and J. Vielmetter and the Protein Expression Center in the Beckman Institute at Caltech. This work was supported by NIH grant P01-Al138398-S1 (M.C.N., C.M.R. and P.J.B.) and 2U19AI111825 (M.C.N. and C.M.R.); the Caltech Merkin Institute for Translational Research and P50 Al150464 (P.J.B.), George Mason University Fast Grants (D.F.R. and P.J.B.) and the European ATAC consortium EC 101003650 (D.F.R.); R01-AI091707-10S1 to C.M.R.; R37-Al64003 to P.D.B.; R01Al78788 to T. Hatziioannou; The G. Harold and Leila Y. Mathers Charitable Foundation to C.M.R. Electron microscopy was performed in the Caltech Beckman Institute Resource Center for Transmission Electron Microscopy (directors: S. Chen and A. Malyutin). C.G. was supported by the Robert S. Wennett Post-Doctoral Fellowship, in part by the National Center for Advancing Translational Sciences (National Institutes of Health Clinical and Translational Science Award programme, grant UL1 TR001866) and by the Shapiro-Silverberg Fund for the Advancement of Translational Research. P.D.B. and M.C.N. are investigators of the Howard Hughes Medical Institute.

Author contributions D.F.R., P.D.B., P.J.B., T. Hatziioannou, C.M.R. and M.C.N. conceived, designed and analysed the experiments. D.F.R., M. Caskey and C.G. designed clinical protocols. F.M., J.C.C.L., Z.W., A.C., M.A., C.O.B., S.F., T. Hägglöf, C.V., K.G., F.B., S.T.C., P.M., H.H., L.N., F.S Y.W., H.-H.H., E.M., A.W.A., K.E.H.-T., N.K. and P.R.H. carried out experiments. A.G. and M. Cipolla 


\section{Article}

produced antibodies. C.O.B., J.E.P. and E.W. produced SARS-CoV-2 proteins. A.H., R.G.K., J.H K.G.M., C.G. and M. Caskey recruited participants and executed clinical protocols. R.P., J.D., M.P. and I.S. processed clinical samples. C.O.B. performed negative-stain electron-microscopy analysis. T.Y.O., A.P.W. and V.R. performed bioinformatics analysis. D.F.R., P.D.B., P.J.B., T.

Hatziioannou, C.M.R. and M.C.N. wrote the manuscript with input from all co-authors.

Competing interests The Rockefeller University has filed a provisional patent application in connection with this work on which D.F.R. and M.C.N. are inventors (US patent 63/021,387)
Additional information

Supplementary information is available for this paper at https://doi.org/10.1038/s41586-0202456-9.

Correspondence and requests for materials should be addressed to D.F.R., P.J.B., P.D.B., M.C.

or M.C.N.

Peer review information Nature thanks Alice McHardy and the other, anonymous, reviewer(s) for their contribution to the peer review of this work.

Reprints and permissions information is available at http://www.nature.com/reprints. 


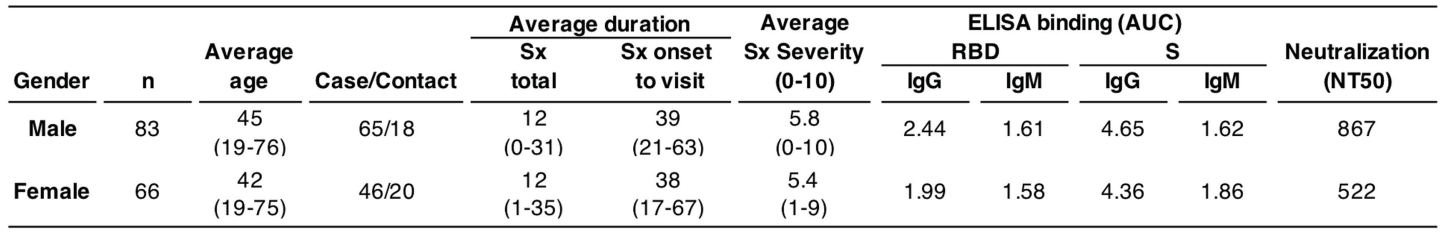


Extended Data Fig. 1 | Clinical correlates. a, Summary of the characteristics of the cohort. Sx, symptoms. b, Age distribution for all men $(n=83)$ and women $(n=66)$ in the cohort. $P=0.2074$.c, Duration of symptoms in days for all men $(n=83)$ and women $(n=66)$ in the cohort. $P=0.8704$. d, Time between symptom onset and plasma collection for all men $(n=83)$ and women $(n=66)$ in the cohort. $P=0.5514$. e, Subjective symptom severity on a scale of $0-10$ for all men $(n=83)$ and women $(n=66)$ in the cohort. $P=0.1888$. $f$, Age distribution for all cases $(n=111)$ and contacts $(n=38)$ in the cohort. $P=0.0305$. g, Duration of symptoms in days for all cases $(n=111)$ and contacts $(n=38)$ in the cohort. $P=0.1241$. h, Time between symptom onset and plasma collection in days for all cases $(n=111)$ and contacts $(n=38)$ in the cohort. $P=0.1589 . \mathbf{i}$, Symptom
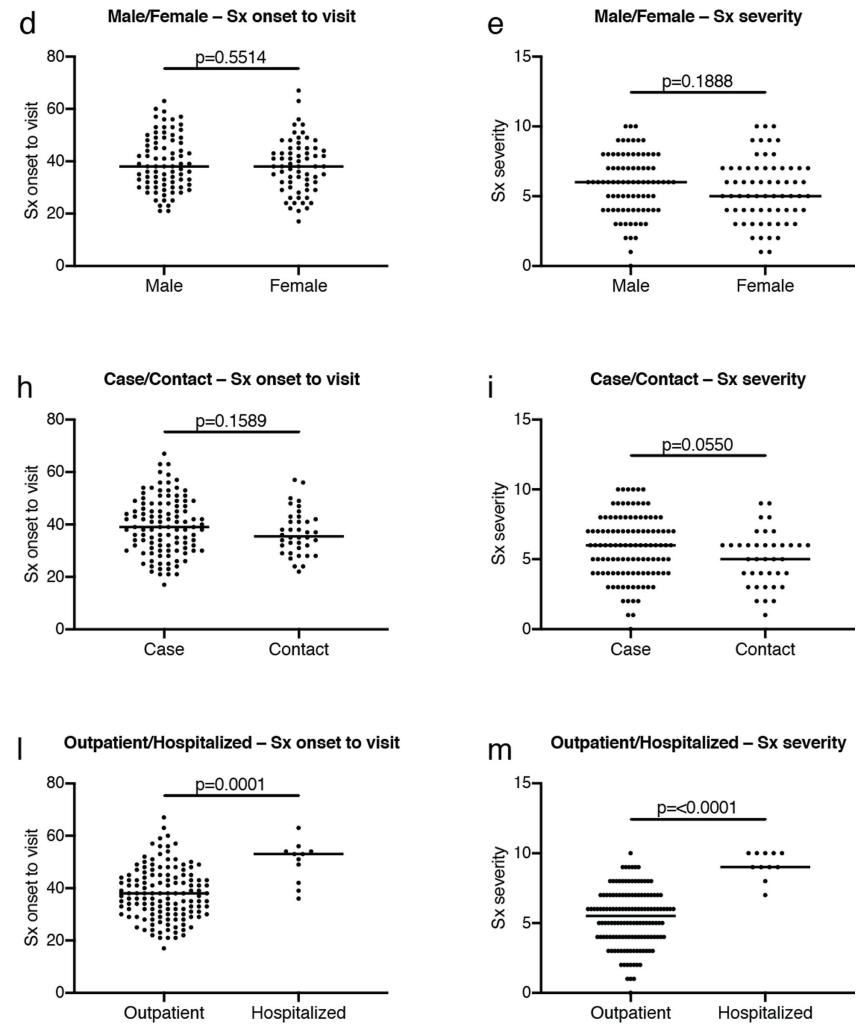

severity for all cases $(n=111)$ and contacts $(n=38)$ in the cohort. $P=0.0550$. j, Age distribution for all outpatient $(n=138)$ and hospitalized $(n=11)$ participants. $P=0.0024 . \mathbf{k}$, Duration of symptoms in days for all outpatient $(n=138)$ and hospitalized $(n=11)$ participants in the cohort. $P<0.0001 .1$, Time between symptom onset and plasma collection in days for all outpatient $(n=138)$ and hospitalized $(n=11)$ participants in the cohort. $P=0.0001$. $\mathbf{m}$, Symptom severity for all outpatient $(n=138)$ and hospitalized $(n=11)$ participants in the cohort. $P<0.0001$. Horizontal bars indicate median values. Statistical significance was determined using two-tailed Mann-Whitney $U$-tests. 


\section{Article}
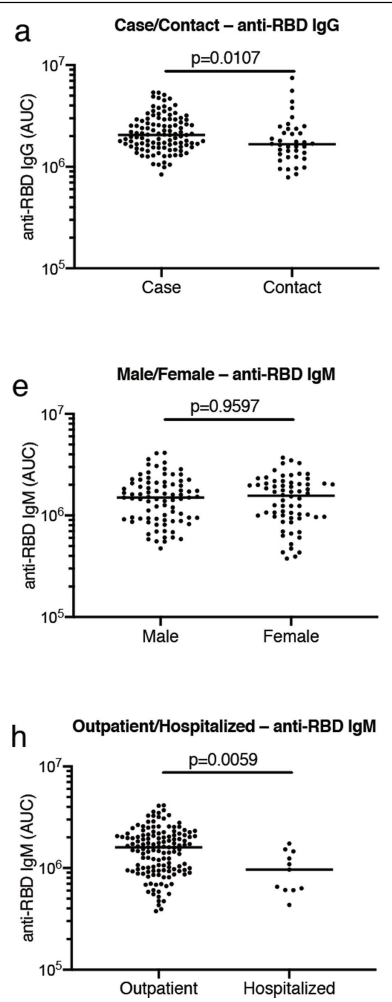
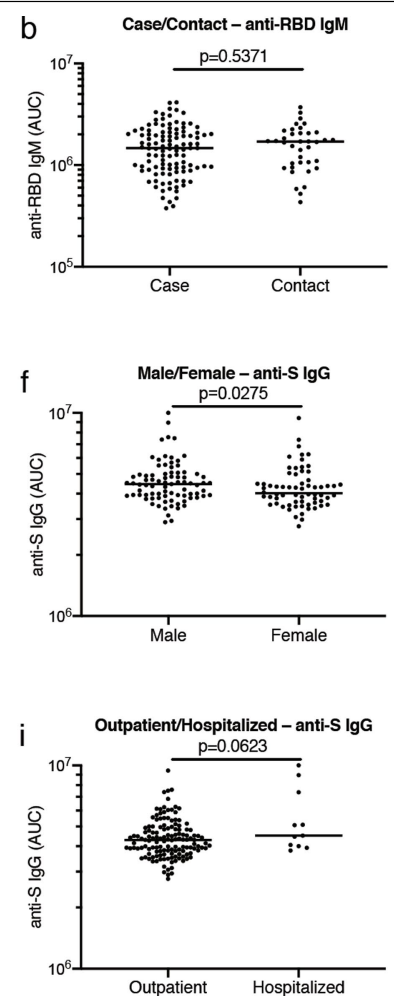

Extended Data Fig. 2 | Clinical correlates of plasma antibody titres.

a, Normalized AUC for IgG anti-RBD for all cases $(n=111)$ and contacts $(n=38)$ in the cohort. $P=0.0107 . \mathbf{b}$, Normalized AUC for IgM anti-RBD for all cases $(n=111)$ and contacts $(n=38)$ in the cohort. $P=0.5371$. , Normalized AUC for IgG anti-S for all cases $(n=111)$ and contacts $(n=38)$ in the cohort. $P=0.0135$. d,

Normalized AUC for IgM anti-S for all cases $(n=111)$ and contacts $(n=38)$ in the cohort. $P=0.7838$. e, Normalized AUC for IgM anti-RBD for all men $(n=83)$ and women $(n=66)$ in the cohort. $P=0.9597 . f$, Normalized AUC for IgG anti-S for all men $(n=83)$ and women $(n=66)$ in the cohort. $P=0.0275$. g, Normalized AUC
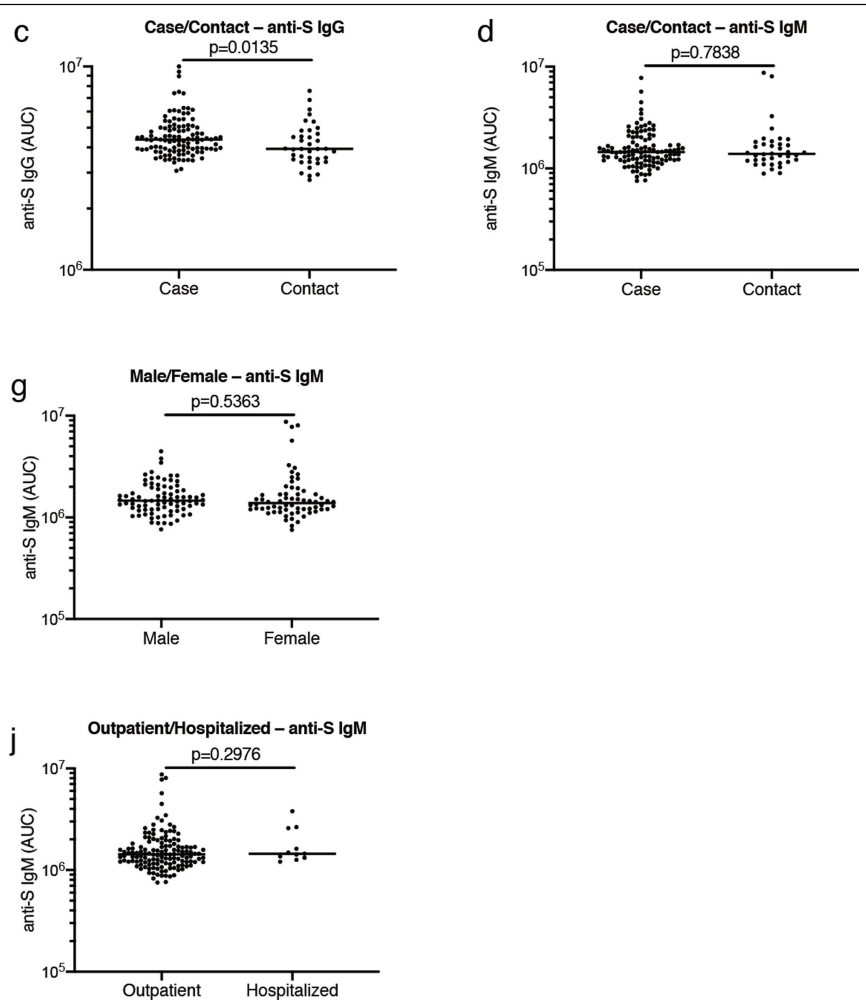

for IgM anti-S for all men $(n=83)$ and women $(n=66)$ in the cohort. $P=0.5363$. h, Normalized AUC for IgM anti-RBD for all outpatient $(n=138)$ and hospitalized $(n=11)$ participants in the cohort. $P=0.0059$. $\mathbf{i}$, Normalized AUC for IgG anti-S for all outpatient $(n=138)$ and hospitalized $(n=11)$ participants in the cohort. $P=0.0623$.j, Normalized AUC for IgM anti-S for all outpatient $(n=138)$ and hospitalized $(n=11)$ participants in the cohort. $P=0.2976$. Horizontal bars indicate median values. Statistical significance was determined using two-tailed Mann-Whitney $U$-tests. 



Extended Data Fig. 3 | Additional clinical correlates of plasma antibody titres. a, Time between symptom onset and plasma collection in days plotted against normalized AUC for IgG anti-RBD. $r=-0.0261, P=0.7533$. b, Time between symptom onset and plasma collection in days plotted against normalized AUC for IgG anti-S. $r=-0.1495, P=0.0697$. c, Time between symptom onset and plasma collection in days plotted against normalized AUC for IgM anti-S. $r=0.1496, P=0.0695$. d, Age plotted against AUC for IgM anti-RBD. $r=0.0172, P=0.8355$. e, Age plotted against normalized AUC for IgG anti-S. $r=0.1523, P=0.0638$.f, Age plotted against normalized AUC for IgM anti-S. $r=0.0565, P=0.4934$. , Duration of symptoms in days plotted against normalized AUC for IgG anti-RBD. $r=0.1525, P=0.0633$. $\mathbf{h}$, Duration of
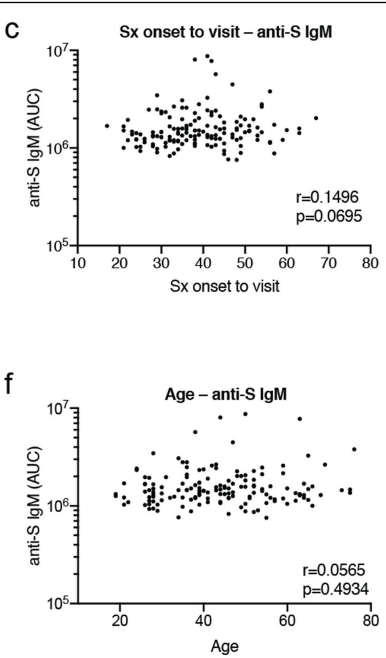

i


$\mathrm{m}$
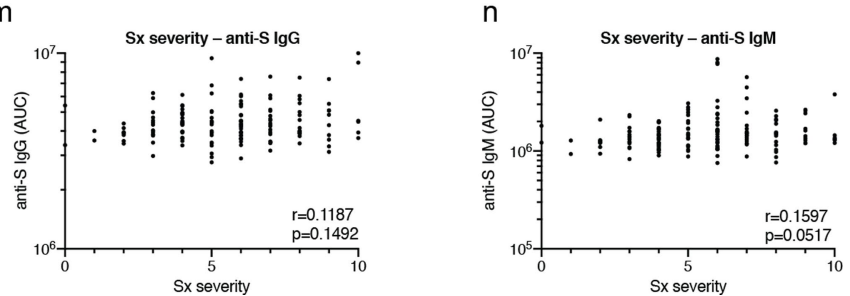

symptoms in days plotted against normalized AUC for IgM anti-RBD. $r=-0.3187, P<0.0001$. i, Duration of symptoms in days plotted against normalized AUC for IgG anti-S. $r=0.0329, P=0.6904$.j, Duration of symptoms in days plotted against normalized AUC for IgM anti-S. $r=0.0824, P=0.3177$. $\mathbf{k}$, Severity of symptoms plotted against normalized AUC for IgG anti-RBD. $r=0.2679, P=0.0010 .1$, Severity of symptoms plotted against normalized AUC for IgM anti-RBD. $r=-0.1943, P=0.0176 . \mathrm{m}$, Severity of symptoms plotted against normalized AUC for IgG anti-S. $r=0.1187 P=0.1492 . \mathbf{n}$, Severity of symptoms plotted against normalized AUC for IgM anti-S. $r=0.1597, P=0.0517$. All correlations were analysed by two-tailed Spearman's tests; $n=149$. 


\section{Article}

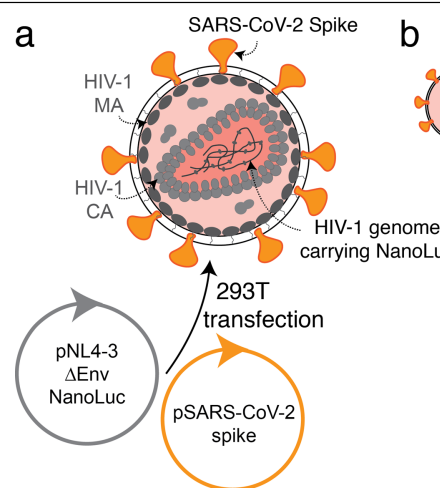

b

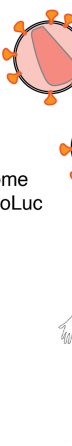

Extended Data Fig. 4 | Diagram of the SARS-CoV-2 pseudovirus luciferase assay. a, Co-transfection of pNL4-3 $\triangle$ Env-nanoluc and pSARS-CoV-2 spike vectors into 293T cells (ATCC) leads to production of SARS-CoV-2 spike-pseudotyped HIV-1 particles (SARS-CoV-2 pseudovirus) carrying the nanoluc gene. b, SARS-CoV-2 pseudovirus is incubated for $1 \mathrm{~h}$ at $37^{\circ} \mathrm{C}$ with plasma or monoclonal antibody dilutions. The virus-antibody mixture is used
C

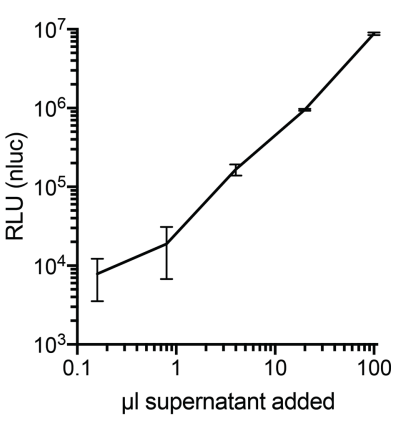

to infect ACE2-expressing 293T cells, which will express nanoluc luciferase upon infection. c, Relative luminescence unit (RLU) reads from lysates of ACE2-expressing 293T cells infected with increasing amounts of SARS-CoV-2 pseudovirus. Data are mean \pm s.d. of triplicates. One representative experiment is shown. 

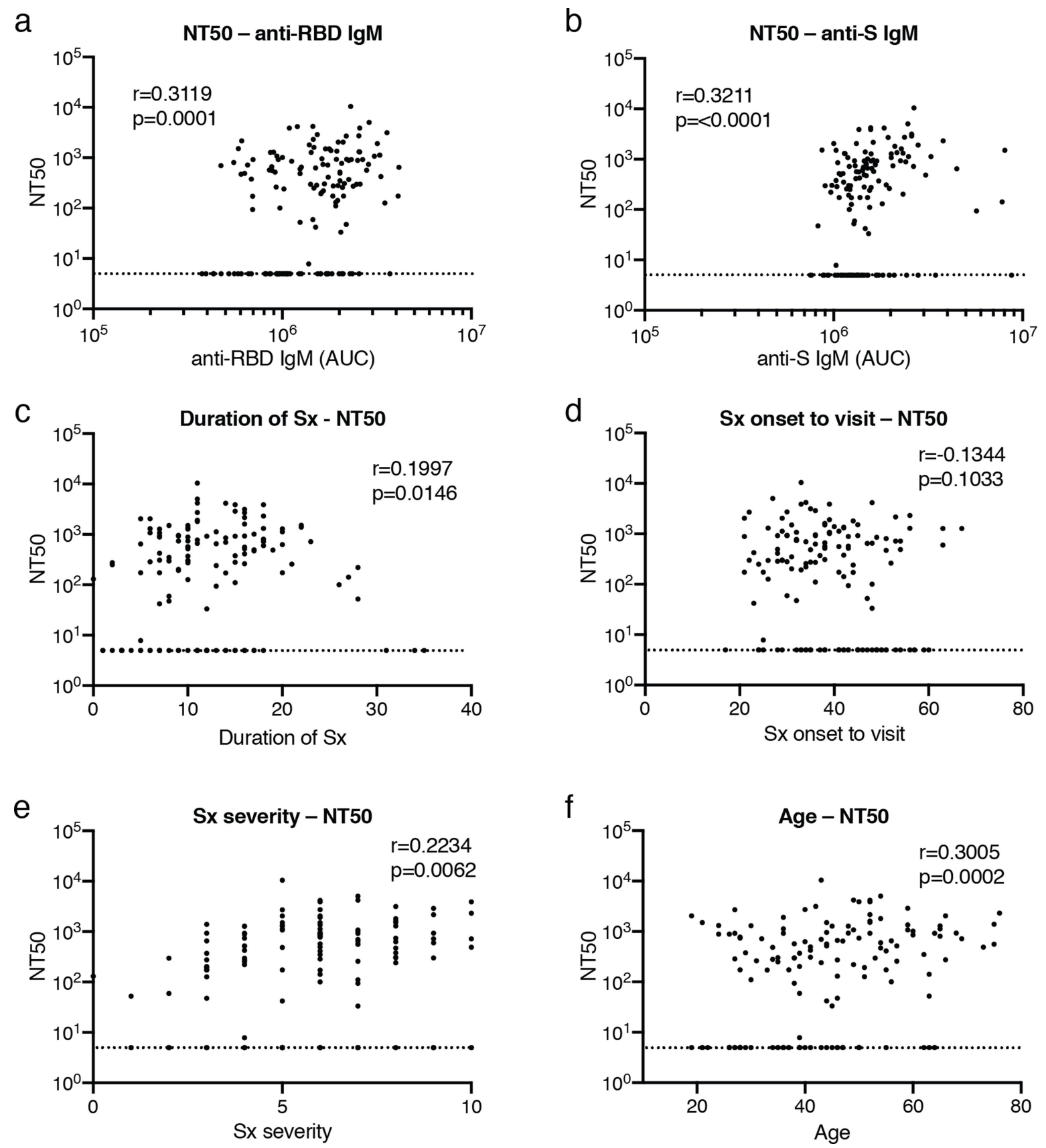

Extended Data Fig. 5 | Clinical correlates of neutralization. a, Normalized AUC for anti-RBD IgM plotted against $\mathrm{NT}_{50} . r=0.3119, P=0.0001$. b, Normalized AUC for anti-S IgM plotted against $\mathrm{NT}_{50} . r=0.3211, P<0.0001$. c, Duration of symptoms in days plotted against $\mathrm{NT}_{50} . r=0.1997, P=0.0146$. d, Time between symptom onset and plasma collection in days plotted against $\mathrm{NT}_{50} . r=-0.1344$, $P=0.1033$.e, Symptom severity plotted against $\mathrm{NT}_{50} . r=0.2234, P=0.0062$.

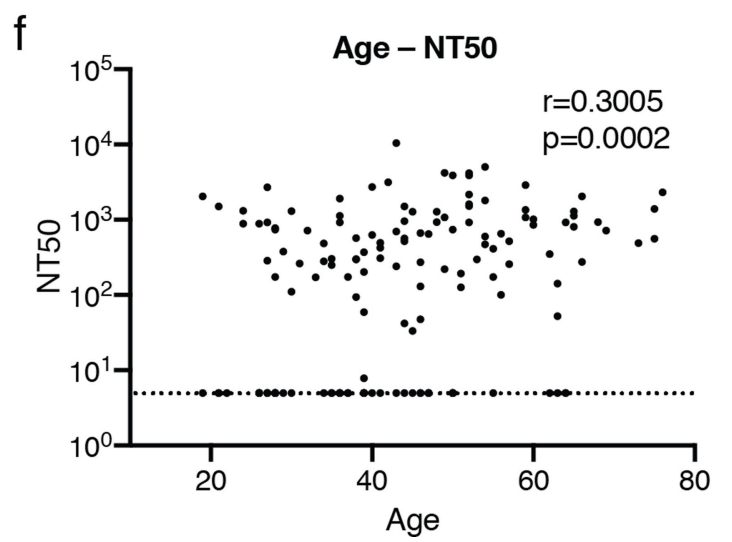

f, Age plotted against $\mathrm{NT}_{50} . r=0.3005, P=0.0002$. All correlations were analysed by two-tailed Spearman's tests; $n=149$. The dotted line $\left(\mathrm{NT}_{50}=5\right)$ represents the lower limit of detection of the pseudovirus neutralization assay. Samples with neutralizing titres below 1:50 were plotted at the lower limit of detection. 
Article

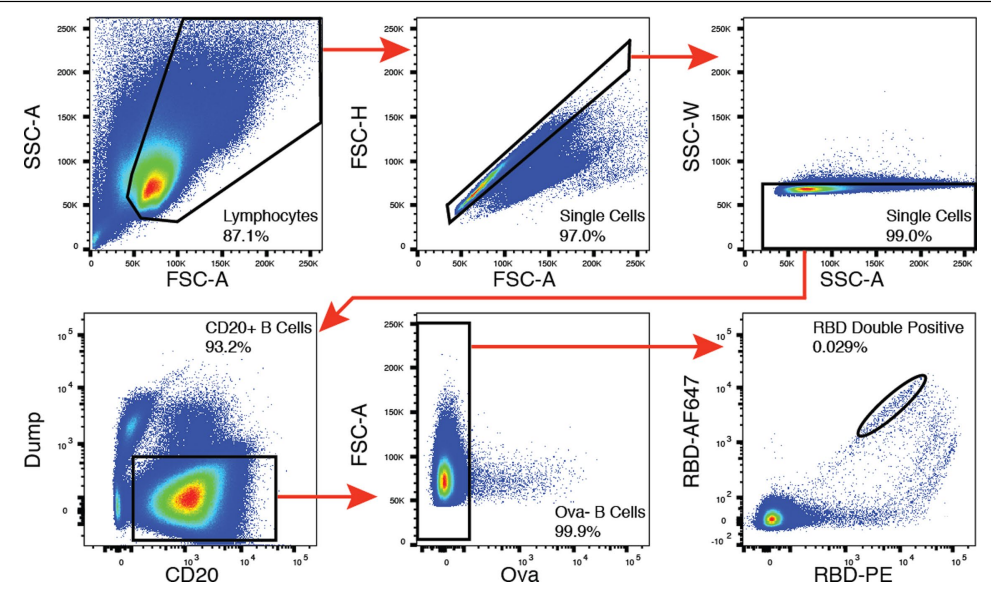

Extended Data Fig. 6 | Flow cytometry. Gating strategy used for cell sorting. Gating was on singlets that were $\mathrm{CD} 2 \mathrm{O}^{+}$and $\mathrm{CD}^{-} \mathrm{CD}^{-} \mathrm{CD} 16^{-} \mathrm{Ova} \mathrm{a}^{-}$. Sorted cells were RBD-PE ${ }^{+}$and RBD-AF647 ${ }^{+}$. 


\section{Heavy frequency (\%)}

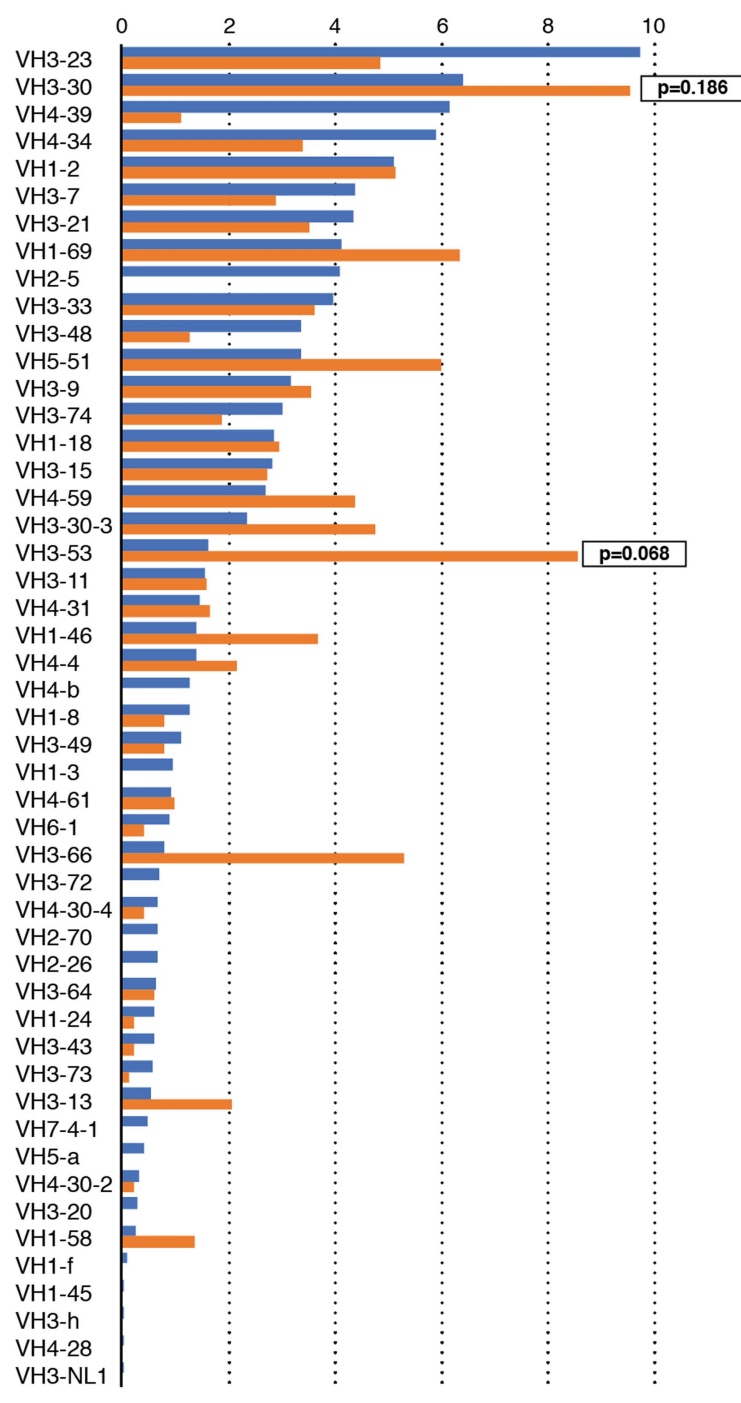

Repertoire

SARS-CoV-2 antibodies

\section{Kappa frequency (\%)}

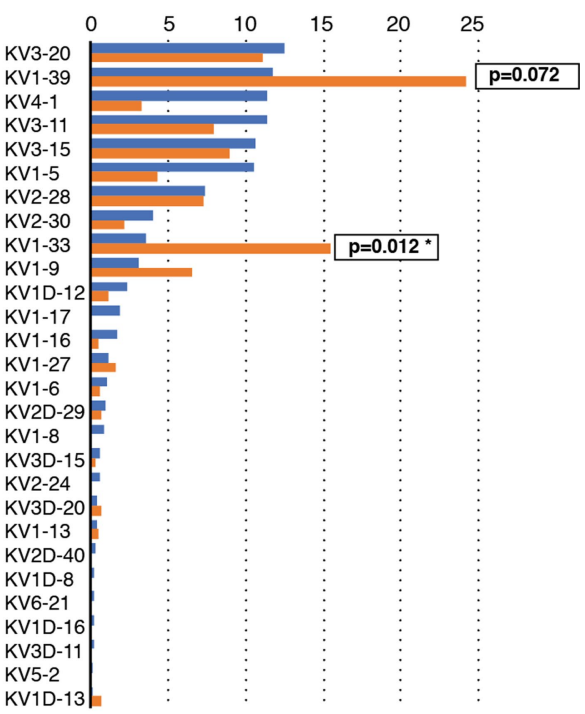

KV1D-13



Extended Data Fig. 7 |Frequency distributions of human V genes. Two-tailed $t$-tests with unequal variance were used to compare the frequency distributions of human V genes of anti-SARS-CoV-2 antibodies from this study to Sequence Read Archive accession SRP010970 ${ }^{41}$. 
a
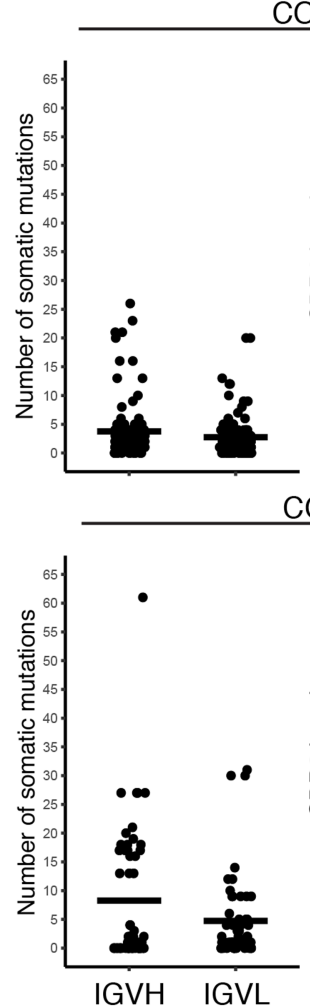

COV107

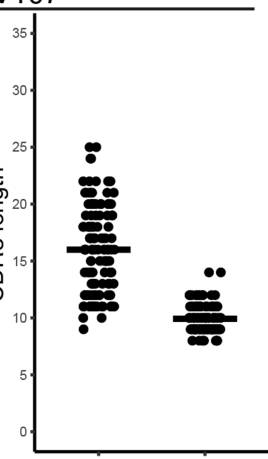

COV57

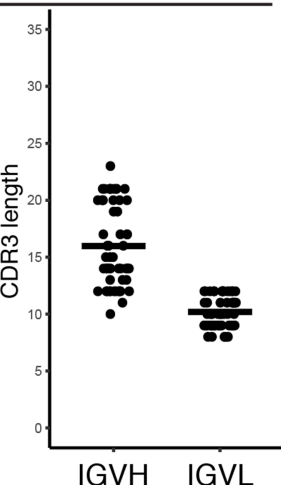

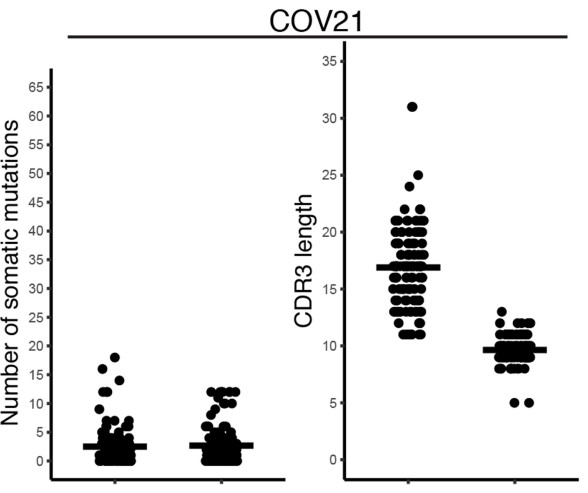

COV72
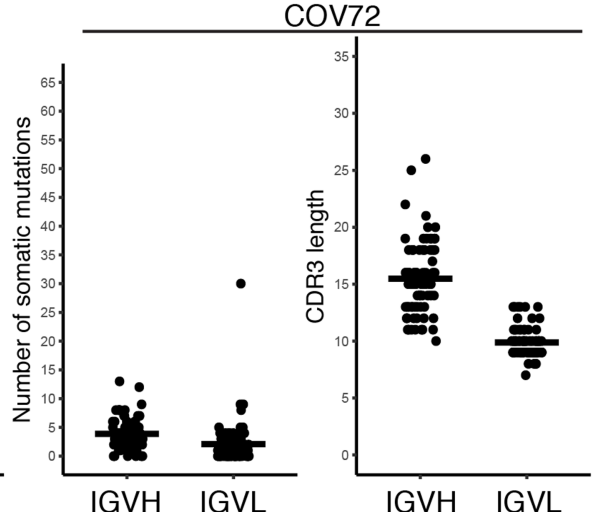

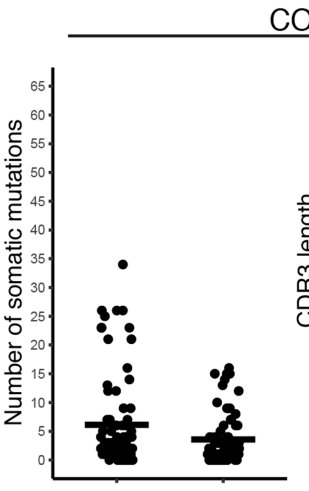

COV47

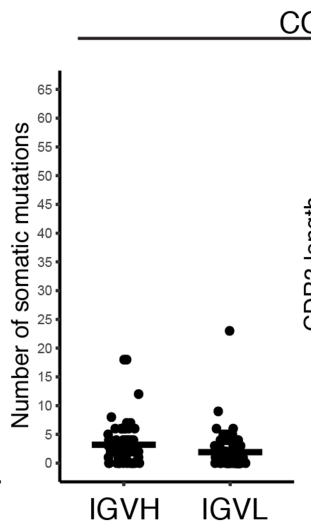

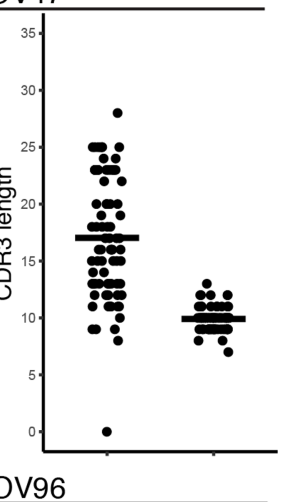

b

Antibodies from all donors

C

Hydrophobicity score (IgH CDR3)
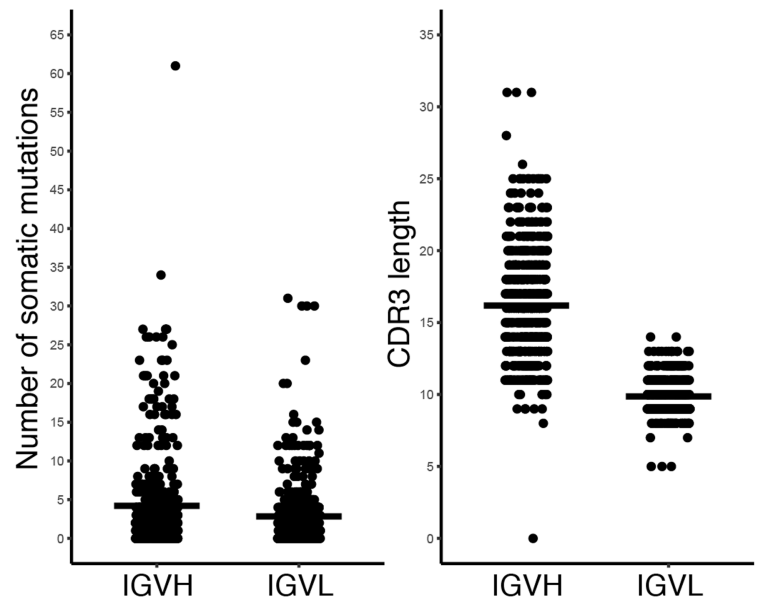

C
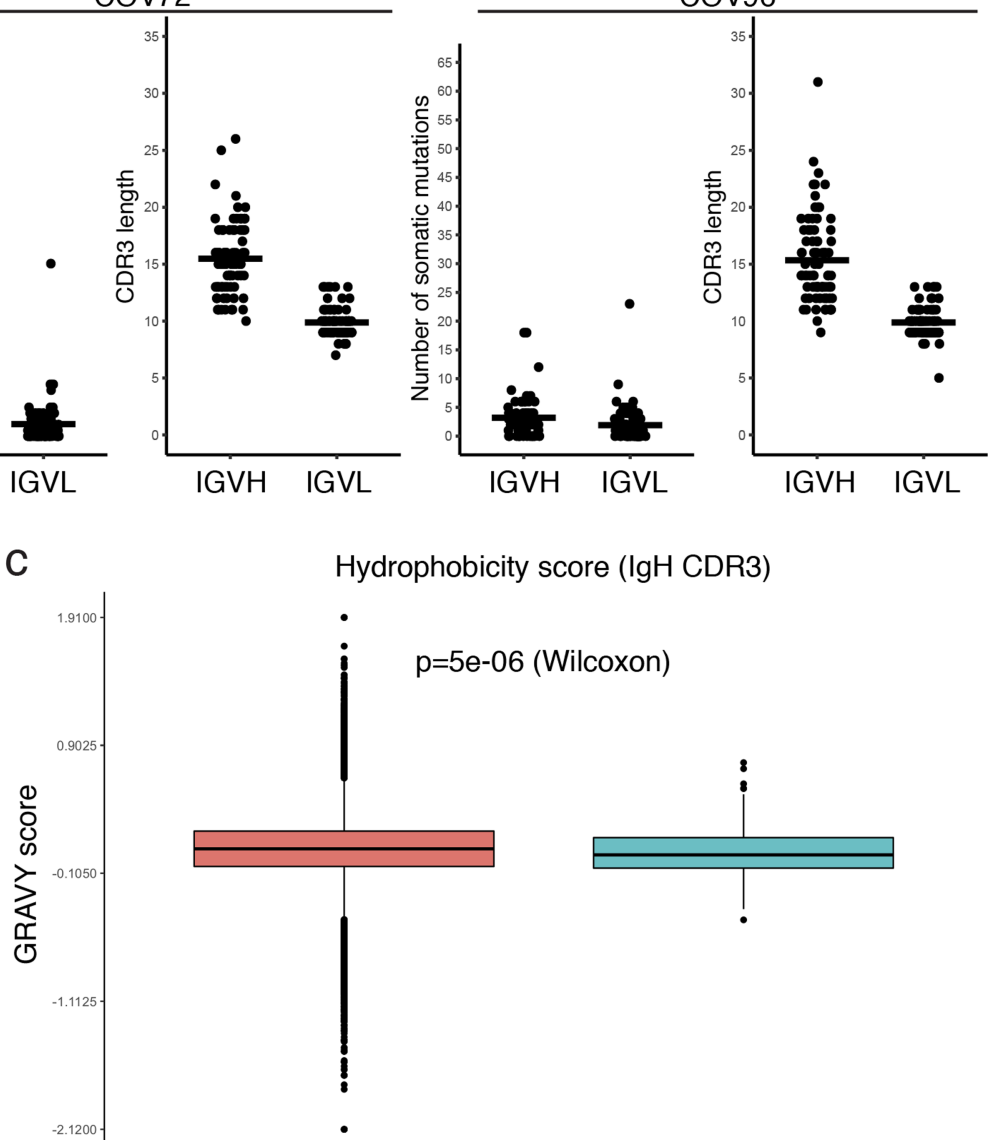

IGVH IGVL

Extended Data Fig. 8 |Analysis of antibody somatic hypermutation and CDR3 length. a, For each individual, the number of somatic nucleotide mutations at the IGVH and IGVL is shown on the left, and the amino acid length of the CDR3s is shown on the right. The horizontal bars indicate the mean. The number of antibody sequences (IGVH and IGVL) evaluated for each participant are $n=118$ (COV107), $n=127$ (COV21), $n=79$ (COV47), $n=54$ (COV57), $n=78$

$$
\begin{array}{ll}
22,654,256 \text { control antibodies } & 533 \text { antibodies } \\
\text { from this study }
\end{array}
$$

(COV72), $n=78$ (COV96). b, Same as in a but for all antibodies combined $(n=534$ for both IGVH and IGVL). c, Distribution of the hydrophobicity GRAVY scores at the IGH CDR3 in antibody sequences from this study compared to a public database (see Methods for statistical analysis). The box limits are at the lower and upper quartiles, the centre line indicates the median, the whiskers are $1.5 \times$ interquartile range and the dots represent outliers. 



Extended Data Fig. 9 |Binding of the monoclonal antibodies to the RBD of SARS-CoV-2 and cross-reactivity to SARS-CoV. a, $\mathrm{EC}_{50}$ values for binding to the RBD of SARS-CoV-2. Average of two or more experiments. $n=89 . \mathbf{b}, \mathbf{c}$, Binding curves (b; representative experiment) and $\mathrm{EC}_{50}$ values (c; mean of two experiments) for binding to the RBD of SARS-CoV. $n=20$ and $n=17$ (excluding

isotype and CR3022) for b and c, respectively. d, e, SARS-CoV pseudovirus neutralization curves and $\mathrm{IC}_{50}$ values.d, Data are mean \pm s.d. of duplicates for one representative experiment. e, Data are the mean of two experiments ( $n=10$, excluding CR3022). Samples with $\mathrm{IC}_{50}$ values above $1 \mu \mathrm{g} \mathrm{ml} \mathrm{I}^{-1}$ were plotted at $1 \mu \mathrm{g} \mathrm{ml}^{-1}$. 


\section{Article}
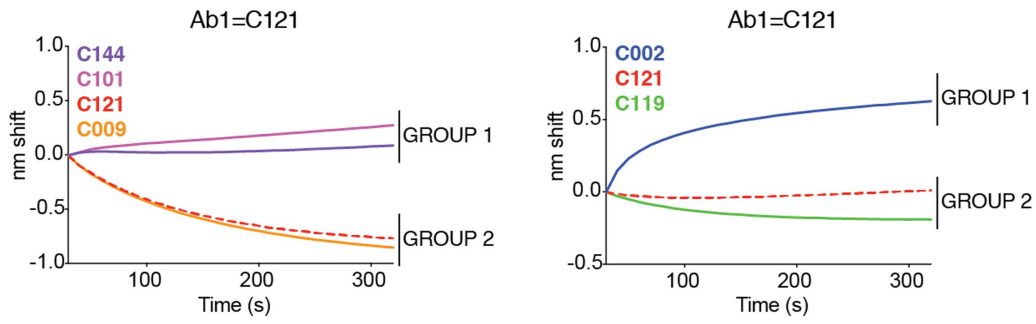

\begin{tabular}{|c|c|c|c|c|c|c|c|}
\hline & \multicolumn{6}{|c|}{ Ab2 } \\
\hline & & C144 & C101 & C002 & C121 & Co09 & C119 \\
\hline \multirow{6}{*}{ Ab1 } & C144 & 0 & 0.2 & $X$ & -0.1 & -0.3 & -0.3 \\
\hline & C101 & -0.1 & 0 & $x$ & -0.1 & -0.2 & -0.1 \\
\hline & C002 & $x$ & $x$ & 0 & -0.2 & $x$ & $x$ \\
\hline & C121 & 0.9 & 1 & 0.7 & 0 & -0.1 & -0.1 \\
\hline & C009 & 1.3 & 1.5 & $x$ & 0.1 & 0 & $X$ \\
\hline & C119 & $X$ & $X$ & 1.1 & -0.2 & $x$ & 0 \\
\hline
\end{tabular}

Extended Data Fig. 10 | Biolayer interferometry experiment. Binding of antibodies C144, C101, C002, C121, C009 and C119. Graphs show second the shift in nanometres after second antibody (Ab2) binding to the antigen in antibody binding to preformed $\mathrm{C} 121$ IgG-RBD complexes. The table displays the presence of the first antibody (Ab1). Values are normalized through the subtraction of the autologous antibody control. 


\section{Reporting Summary}

Nature Research wishes to improve the reproducibility of the work that we publish. This form provides structure for consistency and transparency in reporting. For further information on Nature Research policies, see our Editorial Policies and the Editorial Policy Checklist.

\section{Statistics}

For all statistical analyses, confirm that the following items are present in the figure legend, table legend, main text, or Methods section.

n/a Confirmed

$\square$ The exact sample size $(n)$ for each experimental group/condition, given as a discrete number and unit of measurement

$\square$ A statement on whether measurements were taken from distinct samples or whether the same sample was measured repeatedly

$\square$ The statistical test(s) used AND whether they are one- or two-sided

$\triangle$ Only common tests should be described solely by name; describe more complex techniques in the Methods section.

Х $\square$ A description of all covariates tested

Х $\square$ A description of any assumptions or corrections, such as tests of normality and adjustment for multiple comparisons

A full description of the statistical parameters including central tendency (e.g. means) or other basic estimates (e.g. regression coefficient)

$\bigotimes \square$ AND variation (e.g. standard deviation) or associated estimates of uncertainty (e.g. confidence intervals)

$\varnothing$ For null hypothesis testing, the test statistic (e.g. $F, t, r$ ) with confidence intervals, effect sizes, degrees of freedom and $P$ value noted Give $P$ values as exact values whenever suitable.

$\bigotimes \square$ For Bayesian analysis, information on the choice of priors and Markov chain Monte Carlo settings

Х $\square$ For hierarchical and complex designs, identification of the appropriate level for tests and full reporting of outcomes

$\bigotimes$ Estimates of effect sizes (e.g. Cohen's $d$, Pearson's $r$ ), indicating how they were calculated

Our web collection on statistics for biologists contains articles on many of the points above.

\section{Software and code}

Policy information about availability of computer code

Data collection IRIS by iMedRIS version 11.01 for clinical data collection and management; BD FACSDiva Software Version 8.0.2 for flow sorting; Omega version 5.11 by BMG Labtech for luminometer; Modulus II Microplate Reader User interface version 2.1.0 by TURNER BioSystems; MetaXpress V 6.1.2071 by Molecular Devices; SerialEM automated image acquisition software version 3.7.

Data analysis FlowJo 10.6.2 for FACS analysis; GraphPad Prism 8.4.2; Microsoft Excel 16.36; MacVector 17.5.4 for sequence analysis; Omega MARS V2.10 by BMG Labtech for luminometer; Fortebio Octet Data Analysis Software 8.0; cryoSPARC v2.15 and UCSF chimera version 1.13 .1 for EM analysis; code for sequence analysis can be obtained at https://github.com/stratust/igpipeline.

For manuscripts utilizing custom algorithms or software that are central to the research but not yet described in published literature, software must be made available to editors and reviewers. We strongly encourage code deposition in a community repository (e.g. GitHub). See the Nature Research guidelines for submitting code \& software for further information.

\section{Data}

Policy information about availability of data

All manuscripts must include a data availability statement. This statement should provide the following information, where applicable:

- Accession codes, unique identifiers, or web links for publicly available datasets

- A list of figures that have associated raw data

- A description of any restrictions on data availability

Data are provided in SI Tables 1, 3, 4, 5 and 6; and Figure 3 has associated raw sequencing data (https://github.com/stratust/igpipeline). Databases used in this study include A Public Database of Memory and Naive B-Cell Receptor Sequences (https://datadryad.org/stash/dataset/doi:10.5061/ dryad.35ks2), PDB datasets 6VYB and 6NB6 and Sequence Read Archive SRP010970. 


\section{Field-specific reporting}

Please select the one below that is the best fit for your research. If you are not sure, read the appropriate sections before making your selection. $\bigotimes$ Life sciences $\quad \square$ Behavioural \& social sciences $\quad \square$ Ecological, evolutionary \& environmental sciences

For a reference copy of the document with all sections, see nature.com/documents/nr-reporting-summary-flat.pdf

\section{Life sciences study design}

All studies must disclose on these points even when the disclosure is negative.

Sample size Sample size of 157 individuals was based on how many we were able to recruit for blood donation between April 1 and May 8 , 2020.

Data exclusions 8 contacts (i.e. exposed to SARS-CoV-2 confirmed infected individuals, but themselves not tested by RT-PCR) that did not develop symptoms were excluded from further analyses as it is possible that they were not infected. Exclusion criteria were not pre-established.

Replication All experiments successfully repeated at least twice.

Randomization This is not relevant as this is an observational study.

Blinding This is not relevant as this is an observational study.

\section{Reporting for specific materials, systems and methods}

We require information from authors about some types of materials, experimental systems and methods used in many studies. Here, indicate whether each material, system or method listed is relevant to your study. If you are not sure if a list item applies to your research, read the appropriate section before selecting a response.

Materials \& experimental systems Methods

n/a $\mid$ Involved in the study

$\square \bigotimes$ Antibodies

$\square \bigotimes$ Eukaryotic cell lines

\ $\square$ Palaeontology and archaeology

$\bigotimes \square$ Animals and other organisms

$\mathrm{n} / \mathrm{a}$ Involved in the study

$\square$ \uman research participants

\ $\square$ Clinical data

$\bigotimes \square$ Dual use research of concern

\section{Antibodies}

Antibodies used

Mouse anti-human CD20-PECy7 (BD Biosciences, 335793), clone L27

Mouse anti-human CD3-APC-eFluro 780 (Invitrogen, 47-0037-41), clone OKT3

Mouse anti-human CD8-APC-421eFluro 780 (Invitrogen, 47-0086-42), clone OKT8

Mouse anti-human CD16-APC-eFluro 780 (Invitrogen, 47-0168-41), clone eBioCB16

Mouse anti-human CD14-APC-eFluro 780 (Invitrogen, 47-0149-4), clone 61D3

Peroxidase Goat Anti-Human IgG Jackson Immuno Research 109-036-088

Peroxidase Goat Anti-Human IgM Jackson Immuno Research 109-035-129

Rabbit polyclonal anti-SARS-CoV-2 nucleocapsid antibody (catalog no. GTX135357; GeneTex)

Goat anti-rabbit AlexaFluor 594 (catalog no. A-11012; Life Technologies)

Anti-Zika virus monoclonal antibody Z021 (Robbiani et al, Cell 2017) used as isotype control

Validation

The human monoclonal antibody Z021, which binds to the Envelope Domain III of the Zika virus, was previously reported and validated (PMID: 31413072). No validation statements for the other antibodies that are commercially available.

\section{Eukaryotic cell lines}

Policy information about cell lines

Cell line source(s)

293T (ATCC CRL-11268)

293TAce2 (derived from 293T); new cell line generated in this study

VeroE6 (ATCC CRL-1586)

Expi293F (ThermoFisher cat. A14527) 
Huh 7.5 (a derivative of Huh 7) was generated in the Laboratory of Virology and Infectious Disease, Rockefeller University (Dr. Charles Rice)

Authentication

Mycoplasma contamination

Commonly misidentified lines

(See ICLAC register)
Not authenticated after purchase, with the exception of the Huh 7.5 cells (authenticated by Genetica Cell Line Testing)

The cells were checked for mycoplasma contamination by Hoechst staining or MycoAlert Kit from Lonza.

\section{Human research participants}

\section{Policy information about studies involving human research participants}

Population characteristics

We enrolled 83 males and 66 females with an average age of 45 and 42, respectively. Eligible participants were adults aged 18-76 years who were either diagnosed with SARS-CoV-2 infection by RT-PCR and were free of symptoms of COVID-19 for at least 14 days (cases), or who were close contacts (e.g., household, co-workers, members of same religious community) with someone who had been diagnosed with SARS-CoV-2 infection by RT-PCR and were free of symptoms suggestive of COVID-19 for at least 14 days (contacts). Exclusion criteria included presence of symptoms suggestive of active SARS-CoV-2 infection, or hemoglobin $<12 \mathrm{~g} / \mathrm{dL}$ for males and $<11 \mathrm{~g} / \mathrm{dL}$ for females.

Recruitment

Study participants were recruited at the Rockefeller University Hospital in New York from April 1 through May 8, 2020. Most study participants were residents of the Greater New York City tri-state region and were enrolled sequentially according to eligibility criteria. Participants were first interviewed by phone to collect information on their clinical presentation, and subsequently presented to the Rockefeller University Hospital for a single blood sample collection. The requirement for participants to be free of symptoms for at least 14 days might have favoured enrollment of participants that developed mild COVID-19 courses of infection during the first weeks of recruitment.

Ethics oversight

The Rockefeller University Institutional Review Board (1230 York Avenue, box 330, New York, NY 10065). Protocol DRO-1006 approved on February 6, 2020.

Note that full information on the approval of the study protocol must also be provided in the manuscript.

\section{Flow Cytometry}

Plots

Confirm that:

\The axis labels state the marker and fluorochrome used (e.g. CD4-FITC).

\The axis scales are clearly visible. Include numbers along axes only for bottom left plot of group (a 'group' is an analysis of identical markers).

$\bigotimes$ All plots are contour plots with outliers or pseudocolor plots.

$\bigotimes$ A numerical value for number of cells or percentage (with statistics) is provided.

\section{Methodology}

Sample preparation

Whole blood samples were obtained from study participants recruited through Rockefeller University Hospital. Peripheral blood mononuclear cells (PBMCs) were separated by Ficoll gradient centrifugation. Prior to sorting, PBMCs were enriched for B cells using a Miltenyi Biotech pan B cell isolation kit (cat. no. 130-101-638) and LS columns (cat. no. 130-042-401).

Instrument

FACS Aria III (Becton Dickinson)

Software

Cell population abundance

Gating strategy
BD FACSDiva Software Version 8.0.2 and FlowJo 10.6.2

Sorting efficiency ranged from $40 \%$ to $66 \%$. This is calculated based on the number of IgG-specific antibody sequences that could be PCR-amplified successfully from single sorted cells from each donor.

Cells were first gated for lymphocytes in FSC-A (x-axis) versus SSC-A (y-axis). We identify single cells in FSC-A versus FSC-H, and then SSC-A versus SSC-W. We then select for CD20+ Dump- B Cells in dump (anti-CD3-eFluro 780, anti-CD16-eFluro 780, anti-CD8-eFluro 780, anti-CD14-eFluro 780, Zombie NIR) versus CD20 (anti-CD20-PE-Cy7); dump-negative was considered to be signal less than 250, and CD20-positive was taken to be signal greater than 100. We then gate for Ova- B cells in FSC-A versus Ova-BV711; Ova-negative was considered to be all cells with signal less than 102. Select for TBEV double-positive cells in TBEV EDIII PE versus TBEV EDIII AlexaFluor 647; this gate was made along the $45^{\circ}$ diagonal, above 103 on both axes. See also Extended Data Figure 6.

$\bigotimes$ Tick this box to confirm that a figure exemplifying the gating strategy is provided in the Supplementary Information. 